Check for updates

Cite this: RSC Adv., 2018, 8, 6013

Received 26th November 2017 Accepted 11th January 2018

DOI: $10.1039 / c 7 r a 12796 k$

rsc.li/rsc-advances

\section{Biocompatible tumor-targeting nanocomposites based on CuS for tumor imaging and photothermal therapy}

\author{
Li Liang, $\uparrow$ Shuwen Peng, $\uparrow$ Zhenwei Yuan, Chen Wei, Yuanyuan He, Jinrong Zheng, \\ Yueqing Gu and Haiyan Chen (iD *
}

\begin{abstract}
Active targeting of tumor receptors is a significant approach for cancer diagnosis. Additionally, development of photothermal agents for photothermal therapy (PTT) has attracted great interest in the field of nanomedicine. In the present study, copper sulfide (CUS) nanoparticles capped with bovine serum albumin (BSA), named CuS@BSA, was synthesized by a convenient method. Then, the near-infrared (NIR) fluorescence probe MBA and the tumor-targeting ligand cyclic RGD were further conjugated on the surface of CuS@BSA, and the obtained nanocomposite was named CuS@BSA-MBA-cRGD. The morphology, optical properties, biotoxicity, tumor-targeting capability and in vitro and in vivo tumor inhibition effect were all characterized comprehensively. This nanocomplex demonstrated enhanced photothermal effects and positive tumor targeting. Thus, the nanocomposite CuS@BSA-MBA-CRGD can used as a promising tumor-targeting PTT agent for simultaneous cancer imaging and photothermal treatment.
\end{abstract}

\section{Introduction}

In recent years, near-infrared (NIR) laser-induced photothermal therapy (PTT), has attracted widespread attention. The energy of NIR light is transferred into heat in PTT, which causes tumor cell apoptosis, without damaging normal cells. ${ }^{1-4}$ At present, the development of PTT is mainly dependent on designing novel photothermal agents for transferring light energy to heat effectively. Moreover, PTT combined with tumor imaging facilitates accurate and effective guidance in the treatment of cancer..$^{5-10}$

Theranostics, combining the contrast agents for diagnostics and drugs for therapy, is an effective approach for cancer treatment and risk reduction. ${ }^{6,7}$ Owing to the unique nano-size effect of nano-materials, it can avoid being captured by the reticular endothelial system (RES) and easily penetrate into the tumor vasculars. ${ }^{11,12}$ Therefore, a variety of multifunctional nanoparticles are widely used in cancer diagnosis and treatment. ${ }^{13,14}$ Copper sulfide nanoparticles (CuS NPs) are featured with prominent absorbance and effective heat conversion effect, small particle size, easy modification, and simple preparation. It can be used as a functional component to combine with other diagnostic contrast agents, and then the multi-functional nanocomposite are obtained. ${ }^{10,15,16}$ Hou et al. ${ }^{17}$ reported that a localized diffusion molecular retention (DMR) tumor targeting drug delivery system based on hollow mesoporous CuS NPs

Department of Biomedical Engineering, School of Engineering, China Pharmaceutical University, 24 Tongjia Lane, Gulou District, Nanjing 210009, China. E-mail: chenhaiyan@cpu.edu.cn; Fax: +86-25-83271046; Tel: +86-25-83271080

$\dagger$ Li Liang and Shuwen Peng contributed equally to this work.
(HMCuS NPs) entrapment of anticancer drug. HMCuS NPs possess the property suitable for photoacoustic imaging, which could offer us a theranostic platform. It is also reported that a ultrasmall CuS NPs inside the cavity of ferritin (Fn) nanocages was synthesized by a biomimetic synthesis method..$^{18}$ With the incorporation of radionuclide ${ }^{64} \mathrm{Cu},{ }^{64} \mathrm{CuS}-\mathrm{Fn}$ NCs are served as excellent PET imaging and photothermal therapy agents to achieve superior cancer therapeutic efficiency with good biocompatibility both in vitro and in vivo.

In this paper, bovine serum albumin (BSA) capped CuS NPs were synthesized to further enhance the biocompatibility and stability of CuS NPs. ${ }^{19}$ Integrin $\alpha_{v} \beta_{3}$, a cell-adhesion molecule that is overexpressed on both proliferating tumor vasculature and certain tumors cells $\mathrm{s}^{20}$ was chosen as the molecular target in this study. Cyclic arginine-glycine-aspartic acid (cRGD) peptide is a potent integrin $\alpha_{v} \beta_{3}$ antagonist and has selective affinity for $\alpha_{v} \beta_{3}$. Followed by coupling with NIR probe (MBA) and the tumor targeting ligand cRGD, the functional nanocomposites (CuS@BSAMBA-cRGD) were obtained. First, the optical properties, photothermal effect and cytotoxicity of CuS@BSA-MBA-cRGD were all evaluated. Then, in vivo dynamics and tumor-targeting capability of CuS@BSA-MBA-cRGD were all investigated in normal mouse and tumor-bearing mouse models. Finally, the tumor inhibition effect was evaluated in cell and animal levels.

\section{Materials and methods}

\subsection{Materials}

Thioglycolic acid (TGA), $\mathrm{CuCl}_{2} \cdot 2 \mathrm{H}_{2} \mathrm{O}$, sodium hydroxide $(\mathrm{NaOH})$, anhydrous ethanol (AR), 1-ethyl-3-[3-dimethylaminopropyl] 
carbodiimide hydrochloride (EDC) and $\mathrm{N}$-hydroxysuccinimide (NHS) were bought from Sinopharm Chemical Reagent Co. (Shanghai, China). NIR fluorescence probe MBA (MW = 768) was synthesized in our laboratory. CRGD was obtained from Nanjing Peptide Industry Biotechnology Co. (Nanjing, China). Thioacetamide (TAA) were purchased from Aladdin (Shanghai, China). 3-(4,5-Dimethylthialzol-2-yl)-2,5-diphenyltetrazolium bromide (MTT), DMEM medium, fetal bovine serum (FBS), penicillin, streptomycin, dimethyl sulfoxide (DMSO), trypsin-EDTA, bovine serum albumin (BSA), and Hoechst 33342 were purchased from Jiangsu Kaiji Biotechnology Co. (Jiangsu China). Ultrapure millipore water (18.2 MU) was used. All other solvents and reagents used in this study were certified analytical reagent grade.

\subsection{Synthesis of MBA}

Synthesis of 1 . Compound 1 was prepared according to a literature method with some modifications. ${ }^{1}$ To a chilled solution of DMF (7.33 g, $7.7 \mathrm{~mL}, 100 \mathrm{mmol}$ ) in $10 \mathrm{~mL} \mathrm{CH}_{2} \mathrm{Cl}_{2}, \mathrm{POCl}_{3}$ (15.62 g, $101.89 \mathrm{mmol})$ in $10 \mathrm{~mL} \mathrm{CH}_{2} \mathrm{Cl}_{2}$ was added dropwise under an ice bath. After $30 \mathrm{~min}$, cyclohexanone ( $5 \mathrm{~g}, 50.95 \mathrm{mmol}$ ) in $10 \mathrm{~mL} \mathrm{CH}_{2} \mathrm{Cl}_{2}$ was added, and the resulting mixture was stirred at $80{ }^{\circ} \mathrm{C}$ for $4 \mathrm{~h}$. It was then cooled to room temperature and poured into ice water, and kept overnight to obtain compound 1 as a yellow solid powder $\left(5 \mathrm{~g}, 56.9 \%, \mathrm{mp} 121^{\circ} \mathrm{C}\right)$.

${ }^{1} \mathrm{H}-\mathrm{NMR}\left(500 \mathrm{MHz}, \mathrm{DMSO}-d_{6}\right) \delta: 10.86(\mathrm{~s}, 1 \mathrm{H}), 10.15(\mathrm{~s}, 1 \mathrm{H})$, 7.56 (s, 1H), 2.38 (m, 4H), 1.57-1.62 (qui, 2H, $J=5 \mathrm{~Hz}$ ); HRMS (ESI): $(\mathrm{M}+\mathrm{H})^{+}$calcd for $\mathrm{C}_{8} \mathrm{H}_{10} \mathrm{ClO}_{2}, 172.0291$; found 172.0294.

Synthesis of 2. The reaction mixture of 2,3,3-trimethyl-3Hindole ( $2 \mathrm{~g}, 12.48 \mathrm{mmol})$ and 1,3-propane sultone (4.57 g, 37.44 mmol) was stirred at $100{ }^{\circ} \mathrm{C}$ for $15 \mathrm{~h}$ in $15 \mathrm{~mL} \mathrm{CH} \mathrm{CH}_{3} \mathrm{CN}$. After cooling to room temperature, the mixture was added dropwise into ice water diethyl ether solution. The red precipitate was collected and washed with petroleum ether $(30 \mathrm{~mL})$. The crude product was purified by flash chromatography with gradient elution using DCM and methanol (30:1, v/v) as eluent, to obtain compound 2 as a pale purple solid powder (1.82 g, $\left.51.64 \%, \operatorname{mp} 216^{\circ} \mathrm{C}\right)$.

HRMS (ESI): $m / z$, calcd for $\mathrm{C}_{14} \mathrm{H}_{20} \mathrm{NO}_{3} \mathrm{~S} 282.1159(\mathrm{M}+\mathrm{H})^{+}$, found 282.1163 .

Synthesis of 3. Compound 3 was prepared according the modified literature method. ${ }^{2}$ Compound 2 (1.64 g, $5.81 \mathrm{mmol}$ ) and compound $1(0.50 \mathrm{~g}, 2.90 \mathrm{mmol})$ and sodium acetate $(0.48 \mathrm{~g}, 5.81 \mathrm{mmol})$ were dissolved in $50 \mathrm{~mL}$ of 1-butanol and benzene $(1: 1)$ in a flask equipped with a Dean-Stark trap. The mixture was refluxed for $12 \mathrm{~h}$ to give dark green solution. After the reaction was completed, the solvent was removed under reduced pressure to give the crude product. Purification of the crude product by column chromatography on silica gel using DCM and methanol ( $25: 1, \mathrm{v} / \mathrm{v}$ ) as eluent, to give compound 3 as a green solid powder $\left(1.32 \mathrm{~g}, 64.91 \%, \mathrm{mp} 258{ }^{\circ} \mathrm{C}\right)$.

HRMS (ESI): $m / z$, calcd for $\mathrm{C}_{36} \mathrm{H}_{44} \mathrm{ClN}_{2} \mathrm{O}_{6} \mathrm{~S}_{2} 699.2324(\mathrm{M}+\mathrm{H})^{+}$, found 699.2342 .

Synthesis of 4 . Compound $3(0.2 \mathrm{~g}, 0.28 \mathrm{mmol})$ and 3-mercaptopropionic acid $(0.06 \mathrm{~g}, 0.57 \mathrm{mmol})$ and triethylamine $(0.06 \mathrm{~g}, 0.57 \mathrm{mmol}$ ) were dissolved in $5 \mathrm{~mL}$ of DMF in a flask equipped with a Dean-Stark trap. The mixture was stirred at room temperature for $24 \mathrm{~h}$ to give dark green solution. After the reaction was completed, the solvent was removed under reduced pressure to give the crude product. Purification of the crude product by column chromatography on silica gel using DCM and methanol (10:1, v/v) as eluent, to give compound 4 (MBA) as a green solid powder $\left(0.16 \mathrm{~g}, 75.05 \%, \mathrm{mp} 258{ }^{\circ} \mathrm{C}\right)$. HRMS (ESI): $m / z$, calcd for $\mathrm{C}_{39} \mathrm{H}_{49} \mathrm{~N}_{2} \mathrm{O}_{8} \mathrm{~S}_{3} 769.2646(\mathrm{M}+\mathrm{H})^{+}$, found 769.2659 .

\subsection{Synthesis of CuS NPs}

CuS NPs were synthesized according to a previous report with amplification and modification. ${ }^{\mathbf{2 1 , 2 2}}$ Specifically, $\mathrm{CuCl}_{2} \cdot 2 \mathrm{H}_{2} \mathrm{O}$ $(0.25 \mathrm{mmol})$ was dissolved in $100 \mathrm{~mL}$ of ultrapure water and then TGA $(0.5 \mathrm{mmol})$ was added dropwise with rapid stirring. The $\mathrm{pH}$ of the mixed solution was adjusted with $1 \mathrm{M} \mathrm{NaOH}$ solution to 9.0. Thioacetamide $(0.1 \mathrm{mmol})$ was dissolved in $20 \mathrm{~mL}$ of distilled water and then injected to the above solution. Lastly, the mixture was stirred under a nitrogen atmosphere for $8 \mathrm{~h}$ at $50{ }^{\circ} \mathrm{C}$. The obtained product was freeze-dried and stored at $4{ }^{\circ} \mathrm{C}$ for further study.

\subsection{Synthesis of CuS@BSA}

CuS NPs were capped with BSA using the coupling agents (EDC and NHS) to increase the reaction yield. First, $0.191 \mathrm{~g}$ of EDC and $0.115 \mathrm{~g}$ of NHS (molar ratio of EDC and NHS is $1: 1$ ) were dissolved in $40 \mathrm{~mL}$ of CuS NPs solution, stirred at $40{ }^{\circ} \mathrm{C}$ for $1 \mathrm{~h}$. Subsequently, by centrifuging at $10000 \mathrm{rpm}$ for $5 \mathrm{~min}$, the activated CuS NPs was obtained and re-dissolved in $40 \mathrm{~mL}$ of PBS ( $\mathrm{pH}=7.4$ ) buffer, and then $100 \mathrm{mg}$ of BSA was added into the solution to react overnight. The mixture was purified by centrifuging (12 $000 \mathrm{rpm}, 10 \mathrm{~min}$ ) and re-dissolved in PBS buffer to remove residual BSA, and this process was carried out 3 times. The final product CuS@BSA was stored at $4{ }^{\circ} \mathrm{C}$ for further study.

\subsection{Synthesis of CuS@BSA-MBA}

The NIR fluorescence probe MBA was activated by EDC and NHS and then conjugated with CuS@BSA. First, $7.85 \mathrm{mg}$ of MBA $(0.01 \mathrm{mmol})$ was dissolved in $10 \mathrm{~mL}$ of ultrapure water and incubated with EDC and NHS (molar ratio of EDC and NHS is $1: 1)$ at room temperature for $2 \mathrm{~h}$. Then CuS@BSA was dispersed in $40 \mathrm{~mL}$ of PBS, and $500 \mu \mathrm{L}$ of the activating solution was added dropwise to fully react with BSA for $8 \mathrm{~h}$. Finally, the mixture was collected by centrifuging at $12000 \mathrm{rpm}$ for $10 \mathrm{~min}$ and washed thrice with ultrapure water to remove the physically adsorbed MBA on the surface of BSA. The final product CuS@BSA-MBA was also stored at $4{ }^{\circ} \mathrm{C}$.

\subsection{Synthesis of CuS@BSA-MBA-cRGD}

CuS@BSA-MBA was activated by EDC and NHS (molar ratio of EDC and NHS is $1: 1$ ) in the dark at room temperature for $4 \mathrm{~h}$. Then the supernatant containing EDC and NHS was removed by centrifuging, and the precipitate was re-dissolved in ultrapure water. Subsequently, the aqueous solution containing cRGD was added into the above complex solution to react at room 
temperature for $8 \mathrm{~h}$. Finally, the mixture was collected by centrifugation and was washed thrice with ultrapure water. The obtained product was stored at $4{ }^{\circ} \mathrm{C}$ in the dark.

\subsection{Characterization of CuS@BSA and its nanocomposites}

The morphology and particle size distribution of CuS NPs, CuS@BSA and CuS@BSA-MBA were studied by a FEI Tecnai G2F20 transmission electron microscopy (TEM) with a $200 \mathrm{kV}$ field emission gun. The TEM samples were prepared by drop casting the aqueous solution of CuS@BSA and CuS@BSA-MBA onto 400-mesh carbon-coated copper grids and the excessive solvent was fully evaporated before the TEM investigation. The stability of CuS NPs in deionized water were evaluated by Mastersizer 3000 Laser Particle Size Analyzer (Malvern, British) after 0 day, 1 day, 2 days, 3 days, 4 days and 5 days of preparation, respectively. The absorption spectra of these samples (CuS NPs, MBA, CuS@BSA, CuS@BSA-MBA and CuS@BSAMBA-cRGD) were determined by Lambda 25 UV-Vis spectrophotometer (PE, USA), and the fluorescence spectra of these samples (MBA, CuS@BSA, CuS@BSA-MBA and CuS@BSA-MBAcRGD) were determined by LS-55 fluorescence spectrophotometer (PE, USA). The infrared spectra were measured on an infrared spectrophotometer (Nicolet ECO 2000, USA). The photothermal effects of these samples (CuSNPs, MBA, CuS@BSA, CuS@BSA-MBA and CuS@BSA-MBA-cRGD) were measured by a sensitive digital thermometer (TES-1320, Taiwan) with the irradiation of a laser $\left(808 \mathrm{~nm}, 1.0 \mathrm{~W} \mathrm{~cm}^{-2}\right)$.

\subsection{Cell culture}

The human cell lines U87MG (malignant glioma) and L02 (human normal liver cell line) were obtained from American Type Culture Collection (ATCC, USA). These cell lines were cultured in DMEM medium supplemented with $10 \%(\mathrm{v} / \mathrm{v})$ calf serum, penicillin $\left(100 \mathrm{U} \mathrm{mL}^{-1}\right)$ and streptomycin $(100 \mathrm{mg}$ $\mathrm{mL}^{-1}$ ). Cells were maintained at $37{ }^{\circ} \mathrm{C}$ under a humidified atmosphere containing $5 \% \mathrm{CO}_{2}$.

\subsection{Cytotoxicity of CuS NPs, CuS@BSA-MBA and CuS@BSA- MBA-cRGD}

MTT assessments were carried out to evaluate the cytotoxicity of CuS NPs and their nanocomposites (CuS@BSA, CuS@BSA-MBA and CuS@BSA-MBA-cRGD) on U87MG and L02 cell lines. In detail, the logarithmic growth phase cells were seeded onto 96well plates with each well by adding $100 \mu \mathrm{L}$ of $10 \%$ FBS DMEM medium $\left(2 \times 10^{5}\right.$ cells per well $)$ and subsequently incubated at $37{ }^{\circ} \mathrm{C}$ for $24 \mathrm{~h}$ in a $\mathrm{CO}_{2}$ cultures box. The cells were further maintained at $37{ }^{\circ} \mathrm{C}$ for $24 \mathrm{~h}$ after incubating with CuS NPs, CuS@BSA, CuS@BSA-MBA and CuS@BSA-MBA-cRGD at a wide concentration range from $10 \mu \mathrm{g} \mathrm{mL} \mathrm{mL}^{-1}$ to $200 \mu \mathrm{g} \mathrm{mL}{ }^{-1}$. After that, each well was washed three times with PBS before addition of MTT solution $\left(10 \mu \mathrm{L}, 5.0 \mathrm{mg} \mathrm{mL}^{-1}\right)$ and then incubated for another $4 \mathrm{~h}$.

The medium containing MTT was carefully removed and 150 $\mu \mathrm{L}$ of DMSO was added into each well. The plates were gently shaken for $15 \mathrm{~min}$ at room temperature before the absorbance measurement. The optical density (OD) was measured using a multi-well plate reader at $490 \mathrm{~nm}$. The cell viability was calculated using the following equation: viable cells $(\%)=$ $\left(\mathrm{OD}_{\text {treated }} / \mathrm{OD}_{\text {control }}\right) \times 100 \%$. Where $\mathrm{OD}_{\text {treated }}$ was obtained in the presence of the samples; $\mathrm{OD}_{\text {control }}$ was obtained from the incubation medium.

2.9.1 Anticancer effect of CuS@BSA-MBA-cRGD in vitro. To evaluate the therapeutic effect of CuS@BSA-MBA-cRGD on cell levels, U87MG cell suspension $\left(5 \times 10^{4}\right.$ cells per well) was added to laser confocal fluorescence microscopy (LCFM) culture dishes and incubated at $37^{\circ} \mathrm{C}$ for $24 \mathrm{~h}$. Subsequently, these samples (CuS NPs, CuS@BSA, CuS@BSA-MBA and CuS@BSAMBA-cRGD) were incubated for another $4 \mathrm{~h}$ with the cells. The cells were irradiated by an $808 \mathrm{~nm}$ laser $\left(1.0 \mathrm{~W} \mathrm{~cm}^{-2}\right)$ for $5 \mathrm{~min}$. Finally, the cells were stained by Hoechst for $0.5 \mathrm{~h}$ before bioimaging.

In addition, MTT assessments were carried out to evaluate the inhibition effect on tumor cells. Five groups were named as NIR (single light), CuS NPs + NIR, CuS@BSA + NIR, CuS@BSAMBA + NIR and CuS@BSA-MBA-cRGD + NIR. The cells were cultured for $24 \mathrm{~h}$ before incubating with CuS NPs, CuS@BSA, CuS@BSA-MBA and CuS@BSA-MBA-cRGD for another $4 \mathrm{~h}$ at a wide concentration range from $10 \mu \mathrm{g} \mathrm{mL} L^{-1}$ to $200 \mu \mathrm{g} \mathrm{mL}{ }^{-1}$. Then an $808 \mathrm{~nm}$ NIR laser was utilized to irradiate the cells for 5 min with a power of $1.0 \mathrm{~W} \mathrm{~cm}^{-2}$ and the cells were incubated at $37{ }^{\circ} \mathrm{C}$ with $5 \% \mathrm{CO}_{2}$ for $24 \mathrm{~h}$. Finally, the survival ratio of the cells in different groups were evaluated by MTT assessment.

\subsection{In vivo experiment}

2.10.1 Animal subjects and tumor model. Normal mice were purchased from Yangzhou University Comparative Medical Center (Jiangsu, Yangzhou). All animal experiments were performed in strict accordance with the Animal Management Rules of the Ministry of Health of the People's Republic of China (Document no. 55, 2001) and approved by Animal Care and Use Committee of China Pharmaceutical University (Nanjing, China). The tumor-bearing mouse models were established by subcutaneously injecting EAC tumor cells $(\sim 5 \times$ $\left.10^{6}\right)$ in the mouse armpit. As EAC tumors grew to a diameter around $5 \mathrm{~mm}$, the mice were fixed for NIR fluorescence imaging, infrared thermal imaging and photothermal treatment.

2.10.2 Bio-distribution of based on MBA near infrared fluorescent probe in normal and tumor-bearing mice. Normal mice $(n=5)$ were used for the bio-distribution investigation of MBA, CuS@BSA-MBA and CuS@BSA-MBA-cRGD in vivo. In detail, MBA (0.2 mL, $\left.10 \mu \mathrm{mol} \mathrm{L}^{-1}\right)$, CuS@BSA-MBA (0.2 mL, 20 $\left.\mu \mathrm{g} \mathrm{mL}^{-1}\right)$ and CuS@BSA-MBA-cRGD $\left(0.2 \mathrm{~mL}, 20 \mu \mathrm{g} \mathrm{mL} \mathrm{m}^{-1}\right)$ solution were injected into the mice by tail vein injection. In vivo fluorescence imaging was then performed using NIR fluorescence imaging system equipped with a $765 \mathrm{~nm}$ laser. A series of fluorescence images were collected at $0 \mathrm{~h}, 0.5 \mathrm{~h}, 1 \mathrm{~h}, 2 \mathrm{~h}, 4 \mathrm{~h}, 8 \mathrm{~h}$, $12 \mathrm{~h}$ and $24 \mathrm{~h}$ respectively. The mice were dissected at the corresponding time points ( $1 \mathrm{~h}, 4 \mathrm{~h}, 12 \mathrm{~h}$ and $24 \mathrm{~h}$ ) and the fluorescence images of different organs (heart, liver, spleen, lung, kidney and intestine) were collected to investigate the bio- 
distribution of MBA, CuS@BSA-MBA and CuS@BSA-MBA-cRGD in vivo.

2.10.3 In vivo targeting study of CuS@BSA-MBA-cRGD based on cRGD. The in vivo targeting ability of CuS@BSAMBA-cRGD to tumor was investigated by intravenously injecting CuS@BSA-MBA-cRGD $\left(0.2 \mathrm{~mL}, 10 \mu \mathrm{g} \mathrm{mL}{ }^{-1}\right)$ into the EAC tumor-bearing mice. The NIR fluorescence images were collected at different time points $(0.5 \mathrm{~h}, 1 \mathrm{~h}, 2 \mathrm{~h}, 7 \mathrm{~h}, 9 \mathrm{~h}, 12 \mathrm{~h}, 24$ $\mathrm{h})$. The mice were then dissected at the strongest fluorescence time points $(9 \mathrm{~h})$ and the fluorescence images of the organs (tumor, intestine, kidney, lung, spleen, liver and heart) were collected to investigate the bio-distribution of CuS@BSA-MBAcRGD in various organs and tumor.

2.10.4 In vitro and in vivo infrared thermal imaging. The infrared thermal images of PBS, CuS NPs, CuS@BSA, CuS@BSAMBA and CuS@BSA-MBA-cRGD aqueous solution $\left(200 \mu \mathrm{g} \mathrm{mL}{ }^{-1}\right.$, $\left.750 \mu \mathrm{g} \mathrm{mL}{ }^{-1}\right)$ were collected by an infrared thermal imaging camera (Fluke, USA) after $808 \mathrm{~nm}$ laser $\left(1.0 \mathrm{~W} \mathrm{~cm}^{-2}\right)$ irradiated for different times $(0,1,3,5$ and $8 \mathrm{~min})$. The normal mice were subcutaneously injected in the right lower limb with $50 \mu \mathrm{L}$ of PBS, CuS NPs, CuS@BSA, CuS@BSA-MBA and CuS@BSA-MBAcRGD and the EAC tumor-bearing mice were injected these five samples through an intratumor injection individually. An $808 \mathrm{~nm}$ laser $\left(1.0 \mathrm{~W} \mathrm{~cm}^{-2}\right)$ was employed to perpendicularly irradiate the injected areas of mice respectively. The infrared thermographic maps and changes were recorded by an infrared thermal imaging camera at different time intervals (0 min, $1 \mathrm{~min}, 3 \mathrm{~min}, 5 \mathrm{~min}$ and $8 \mathrm{~min}$ ).

2.10.5 In vivo antitumor effect of CuS@BSA-MBA-cRGD. The EAC tumor-bearing mice were randomly assigned into four groups $(n=5)$ and treated with different injections (saline, NIR, CuS@BSA-MBA + NIR and CuS@BSA-MBA-cRGD + NIR) through the tail vein. The group were injected with saline $(0.2 \mathrm{~mL}$,
$0.154 \mathrm{~mol} \mathrm{~L}^{-1}$ ) as the control group. CuS@BSA-MBA and CuS@BSA-MBA-cRGD were administered in a dose equivalent to the drug concentration of $5.0 \mathrm{mg} \mathrm{kg}^{-1}$. Each tumor-bearing mice were subjected to the tail vein injection once every 2 day, and then were irradiated with an $808 \mathrm{~nm}$ laser (a power density of $1.0 \mathrm{~W} \mathrm{~cm} \mathrm{~cm}^{-2}, 5 \mathrm{~min}$ ) at $9 \mathrm{~h}$ after injection of CuS@BSA-MBA.

The body weight and tumor size of each mouse was monitored every day over 10 days. The tumor volume was calculated as tumor volume $V=(a \times b)^{2} / 2, a$ and $b$ represent the major diameter and minor diameter of the tumor, respectively. The tumor inhibition rate was calculated according to the following equation: inhibition rate $=\left(V_{\mathrm{c}}-V_{\mathrm{t}}\right) / V_{\mathrm{c}} \times 100 \%, V_{\mathrm{c}}$ and $V_{\mathrm{t}}$ represents the mean tumor size of the control and treatment groups, respectively. To further investigate the therapeutic effects of CuS@BSA-MBA-cRGD, the tumors of the four groups were excised for pathological analysis after 10 days of treatment.

\section{Results and discussion}

\subsection{Synthesis and characterization of CuS@BSA and its nanocomposites}

The NIR probe MBA was synthesized according the synthesis route shown in Fig. 1. The nanocomposites (CuS@BSA-MBAcRGD) based on CuS@BSA were synthesized according to the schematic illustration in Fig. 2A. The stabilizer of CuS@BSA, BSA, was coated on the surface of CuS NPs and played a vital role in the synthesis process of CuS@BSA-MBA-cRGD due to its functional carboxyl group and amino group. Herein, the amino groups of BSA were conjugated with the activated carboxyl groups of MBA via NHS/EDC to obtain CuS@BSA-MBA. ${ }^{23}$ Subsequently, the obtained CuS@BSA-MBA was activated with EDC/NHS and reacted with cRGD overnight in the dark to obtain CuS@BSA-MBA-cRGD and then stored at $4{ }^{\circ} \mathrm{C}$. Fig. 2B<smiles>O=C1CCCCC1CCCOCC1=C(Cl)C(=CO)CCC1</smiles>

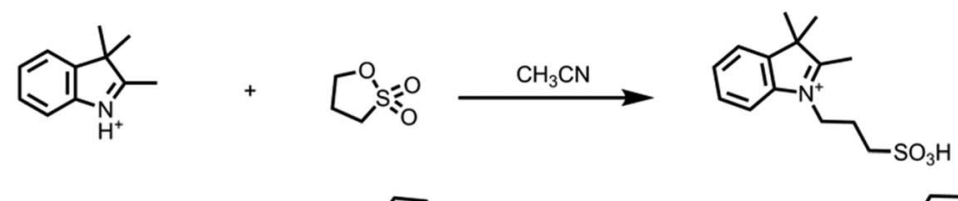
2
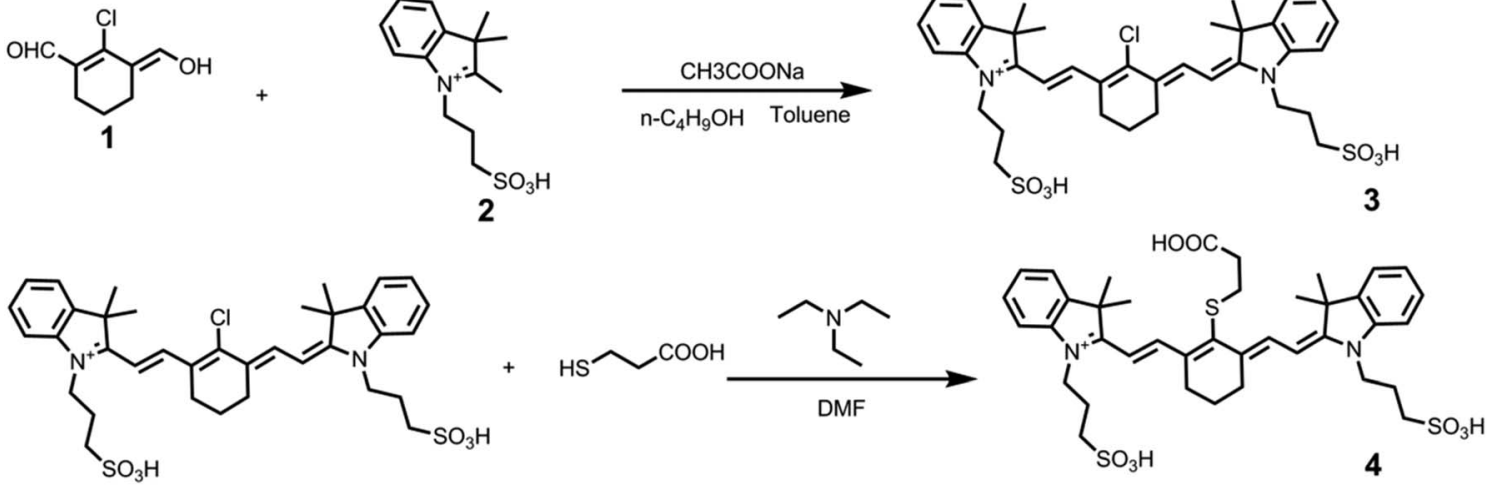

Fig. 1 The synthesis route of MBA. 

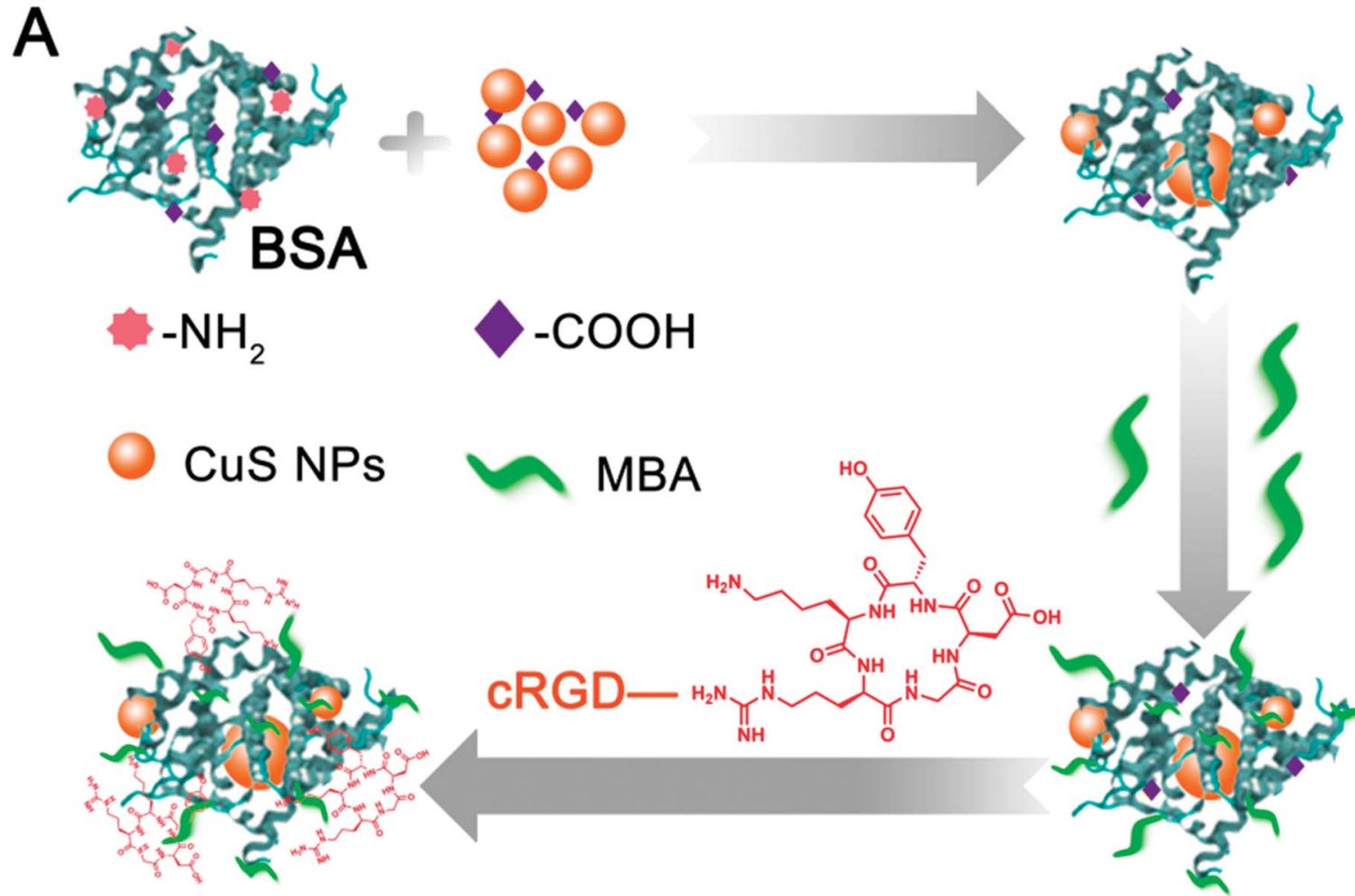

B
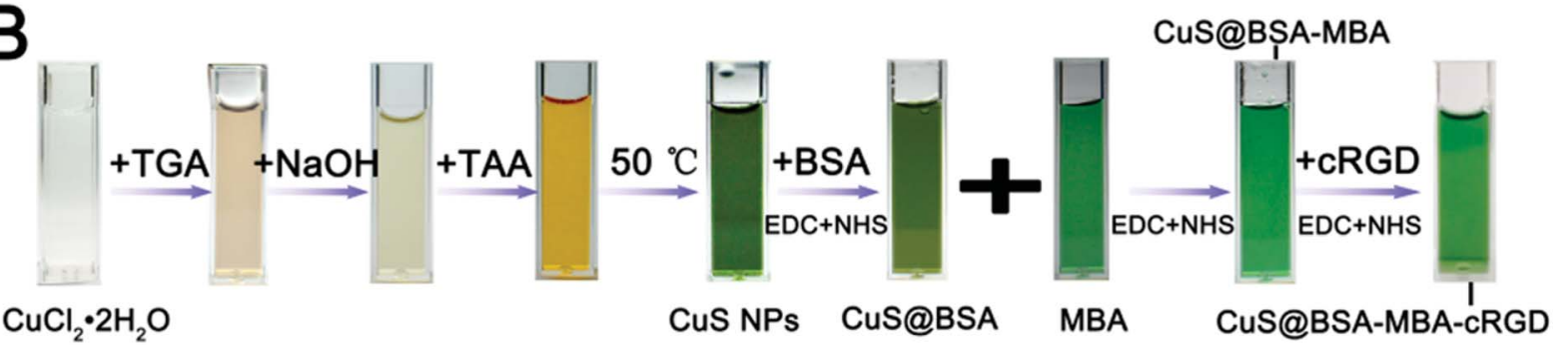

Fig. 2 (A) The diagram for the preparation of CuS@BSA-MBA-CRGD; (B) the real color photos of different samples (CuS NPs, CuS@BSA, MBA, CuS@BSA-MBA, CuS@BSA-MBA-cRGD).

demonstrates the real color photos of different samples (CuS NPs, CuS@BSA, MBA, CuS@BSA-MBA and CuS@BSA-MBAcRGD), which shows that the color of CuS@BSA changed from dark green to bright green after the NIR probe MBA conjugated with CuS@BSA. And the addition of cRGD did not change the color of the solution obviously.

As shown in Fig. 3A, the TEM image indicated spherical shape and homogeneous distribution of CuS nanoparticles. Also, their size distribution in deionized water was studied with 5 days after preparation. The result indicated the favorable stability of CuS nanoparticles due to their constant hydrodiameters in deionized water (Fig. 3D). The TEM images of CuS@BSA and CuS@BSA-MBA were shown in Fig. 3B and C respectively. CuS@BSA and CuS@BSA-MBA are almost spherical shapes and have a homogeneous distribution. Fig. 3E and F show that the mean hydrodynamic diameter of CuS@BSA and CuS@BSA-MBA are $39.8 \pm 4.87 \mathrm{~nm}$ and $51.2 \pm 2.46 \mathrm{~nm}$ respectively, and they all displayed narrow size distribution.

As indicated in Fig. 4A, there is no change for the absorption peak of CuS NPs after coupling with BSA. Both of CuS@BSA-
MBA and CuS@BSA-MBA-cRGD have the same absorption peak at $787 \mathrm{~nm}$, which is corresponding to the characteristic absorption peak of MBA. As shown in Fig. 4B, the fluorescence peak of MBA shifted from $802 \mathrm{~nm}$ to $825 \mathrm{~nm}$ after it coupled with CuS@BSA. The fluorescence peak of CuS@BSA-MBA red shifts slightly to $829 \mathrm{~nm}$ after it conjugated with cRGD, which indicates that both MBA and cRGD are coupled with CuS@BSA by covalent bond rather than simple physical adsorption. After conjugation with CuS@BSA, the $\pi$ electron conjugated system with donor and acceptor groups are extended due to the contribution of the new unsaturated groups formed by the conjugation of MPA and CuS@BSA. Therefore, the fluorescence peak of MBA red-shifted around $20 \mathrm{~nm}$.

The Fourier transfer infrared (FI-IR) spectroscopy of the samples (CuS NPs, CuS@BSA, MBA and CuS@BSA-MBA) are shown in Fig. 5. CuS NPs have a broad band peak at $3448 \mathrm{~cm}^{-1}$, which is corresponding to the deformation vibration of $\mathrm{O}-\mathrm{H}$ on the carboxyl group (Fig. 5A). The band appearing at $1626 \mathrm{~cm}^{-1}$ can be attributed to the characteristic stretching and bending vibration of $\mathrm{C}=\mathrm{O}$ on the carboxyl group. The disappearance of 

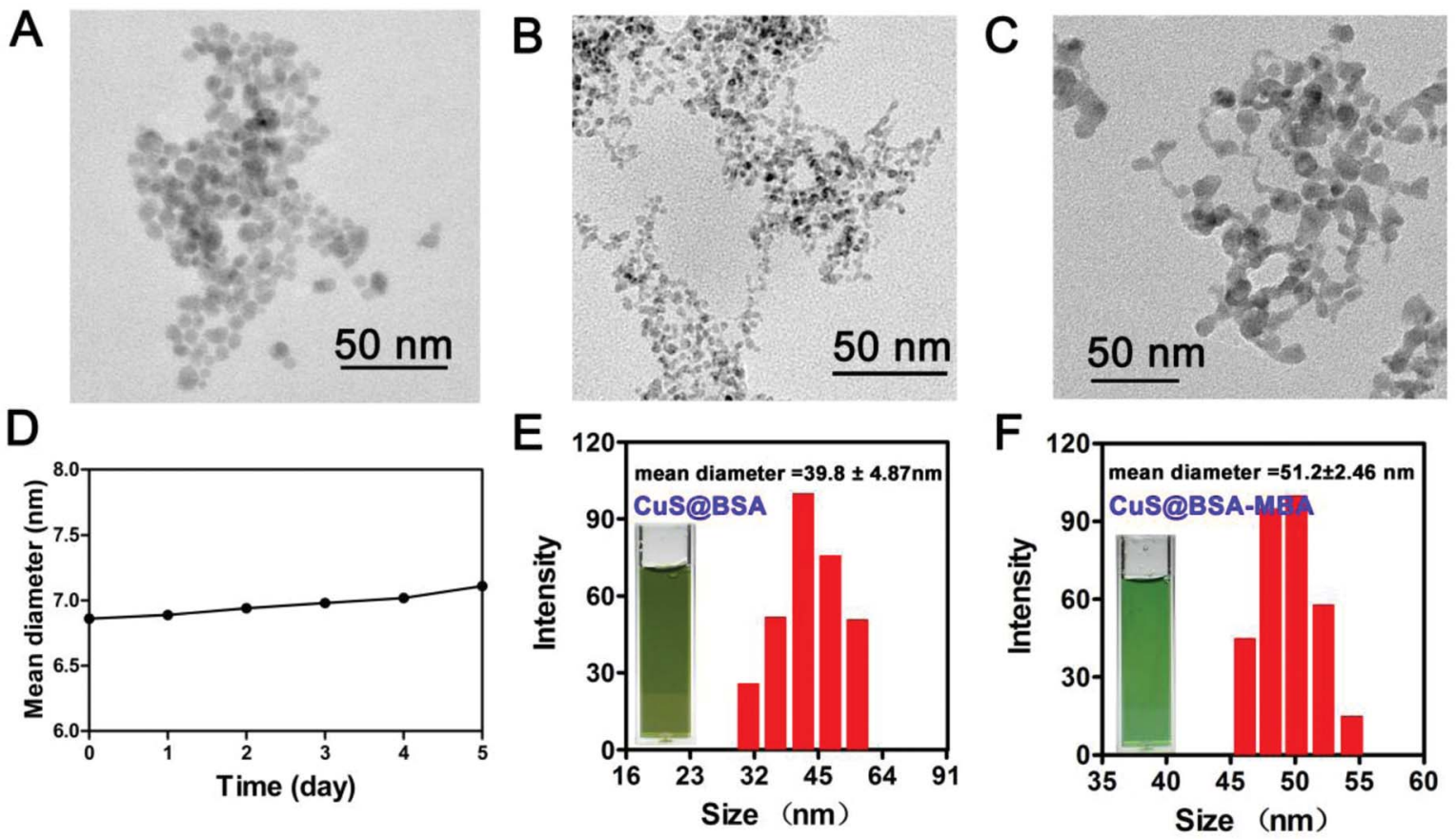

Fig. 3 The TEM image of (A) CuS NPs, (B) CuS@BSA and (C) CuS@BSA-MBA; (D) the plot of the hydrodiameters of CuS NPs as different storage days; TEM images of the hydrodynamic size distribution and the inserted real color pictures of (E) CuS@BSA and (F) CuS@BSA-MBA.

the characteristic band of the carboxyl group in CuS@BSA indicates the formation of new covalent bonding groups. The band centered at $3287 \mathrm{~cm}^{-1}$ is attributed to $\mathrm{N}-\mathrm{H}$ stretching vibration of amide. The characteristic band of $1644 \mathrm{~cm}^{-1}$ is corresponding to $\mathrm{C}=\mathrm{O}$ stretching vibration of amide, which further indicates that BSA is successfully coupled with CuS NPs. Similarly, the FI-IR spectroscopy (Fig. 5B) of MBA shows a moderate peak at $3384 \mathrm{~cm}^{-1}$, corresponding to the deformation vibration of $\mathrm{OH}$ on the carboxyl group. The disappearance of this characteristic band in the FI-IR spectroscopy of
CuS@BSA-MBA also proves that MBA has been successfully covalently linked to CuS@BSA.

In order to investigate the photothermal effect of CuS NPs and their nanocomposites, four different concentrations of these nanoparticles (MBA, CuS NPs, CuS@BSA, CuS@BSA-MBA and CuS@BSA-MBA-cRGD) were irradiated with an $808 \mathrm{~nm}$ laser (a power density of $1.0 \mathrm{~W} \mathrm{~cm}^{-2}, 6 \mathrm{~min}$ ). For different concentrations of MBA, the $2.0 \mathrm{mg} \mathrm{mL}^{-1}$ and $4.0 \mathrm{mg} \mathrm{mL}^{-1}$ concentration curves show a obvious progress of first rise and fall (Fig. 6A). The first rise is due to the strong absorption of MBA
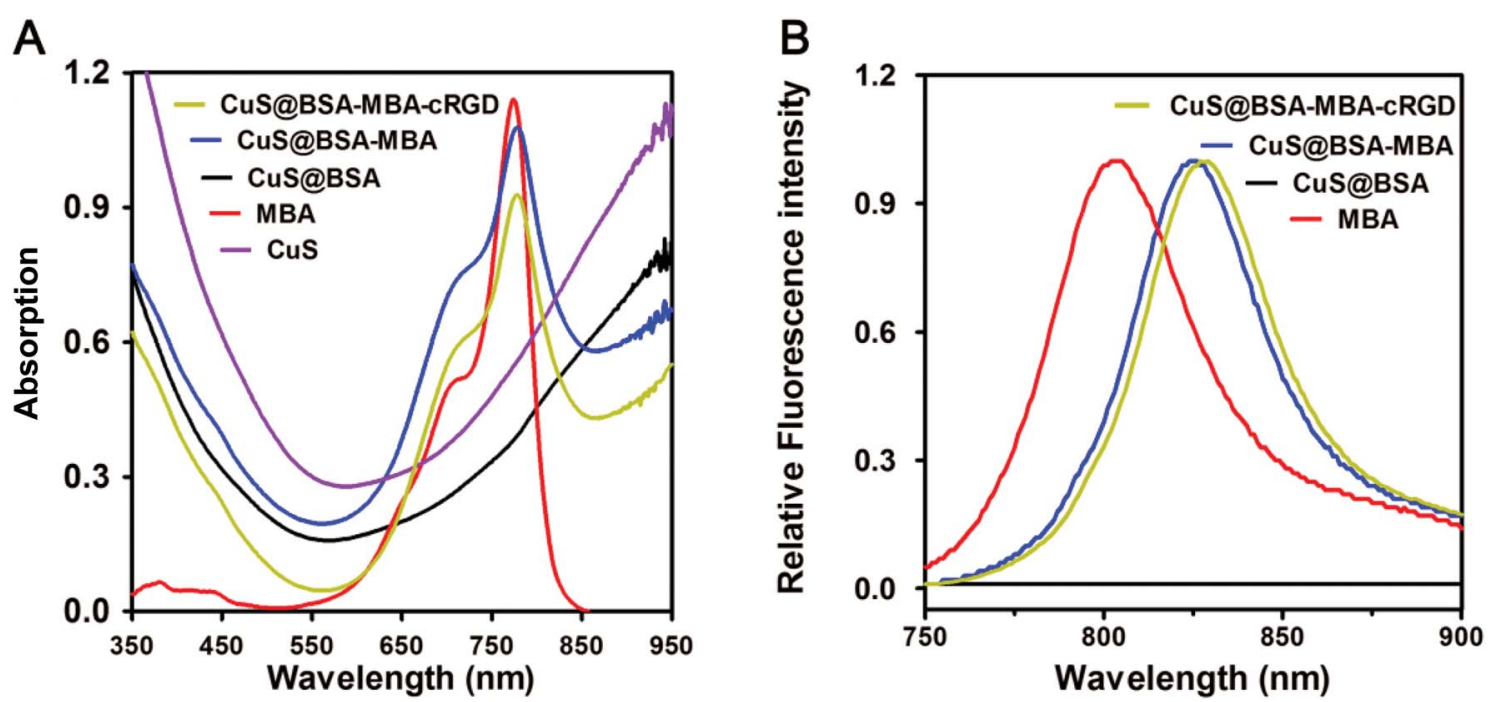

Fig. 4 (A) Absorption spectra of CuS NPs, MBA, CuS@BSA, CuS@BSA-MBA and CuS@BSA-MBA-cRGD; (B) fluorescence spectra of MBA, CuS@BSA, CuS@BSA-MBA and CuS@BSA-MBA-cRGD. 

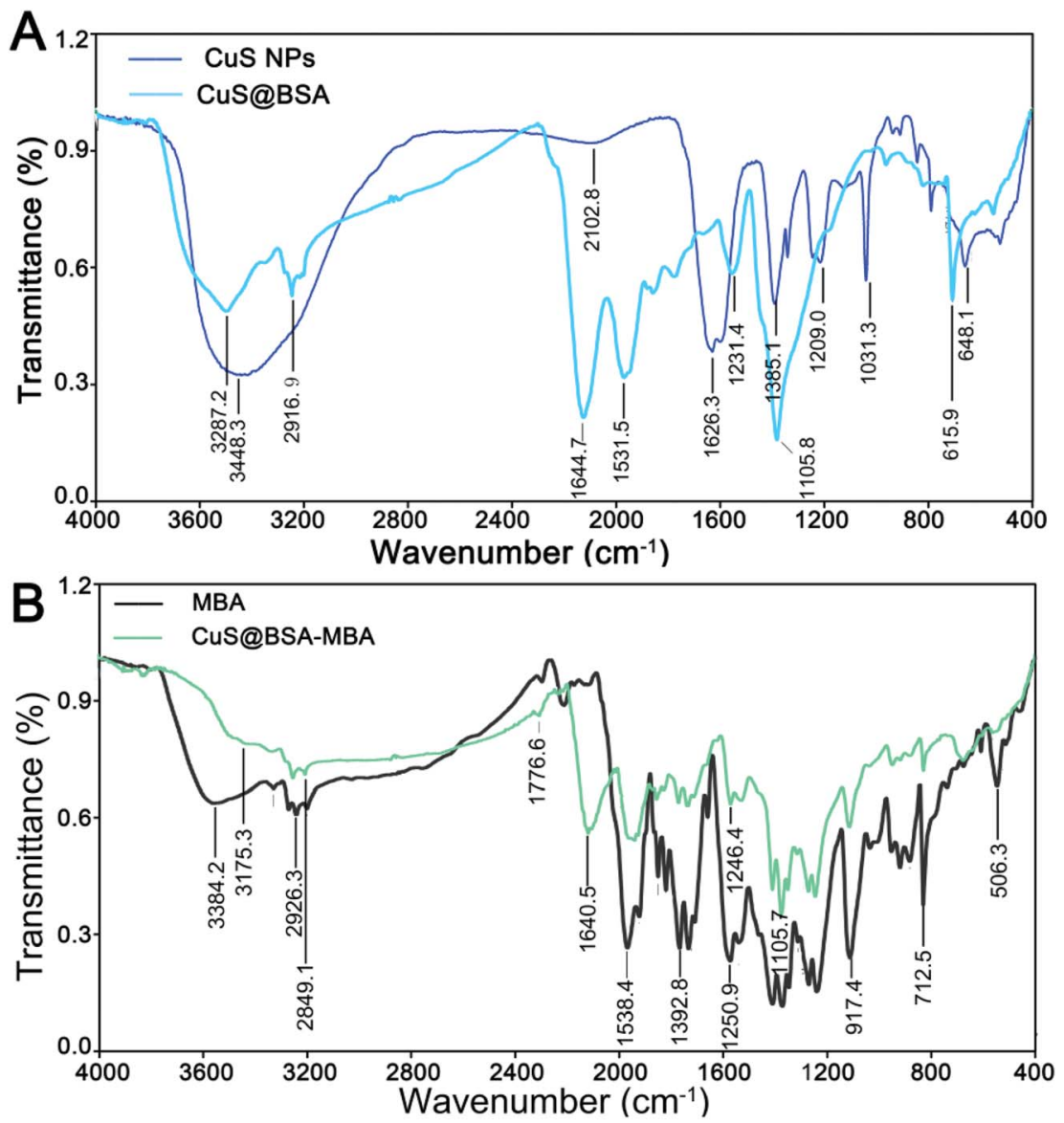

Fig. 5 (A) FI-IR spectroscopy of CuS NPs and CuS@BSA; (B) FI-IR spectroscopy of MBA and CuS@BSA-MBA.
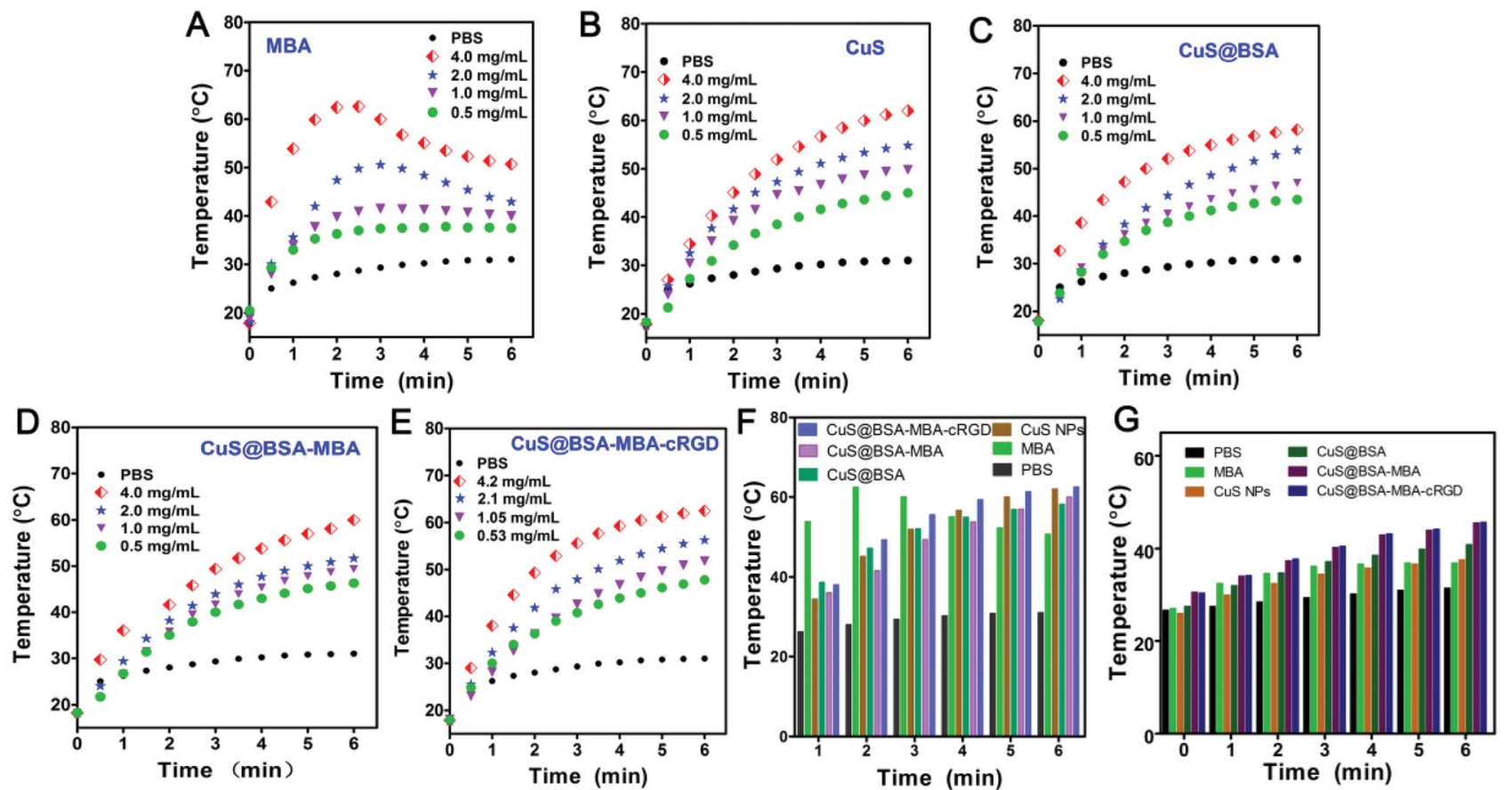

Fig. 6 The photothermal effect of (A) MBA, (B) CuS NPs, (C) CuS@BSA, (D) CuS@BSA-MBA and (E) CuS@BSA-MBA-cRGD at different concentrations $\left(0.5 \mathrm{mg} \mathrm{mL}^{-1}, 1.0 \mathrm{mg} \mathrm{mL}^{-1}, 2.0 \mathrm{mg} \mathrm{mL}^{-1}, 4.0 \mathrm{mg} \mathrm{mL}^{-1}\right.$ ) and irradiation time intervals (1 min to $6 \mathrm{~min}$ ); quantitative comparison of the temperature changing of different samples (PBS, MBA, CuS NPs, CuS@BSA, CuS@BSA-MBA and CuS@BSA-MBA-cRGD) at concentrations of (F) $4.0 \mathrm{mg} \mathrm{mL}^{-1}$ and (G) $200 \mu \mathrm{g} \mathrm{mL}^{-1}$ in different laser irradiation times (1 to $6 \mathrm{~min}$ ). 

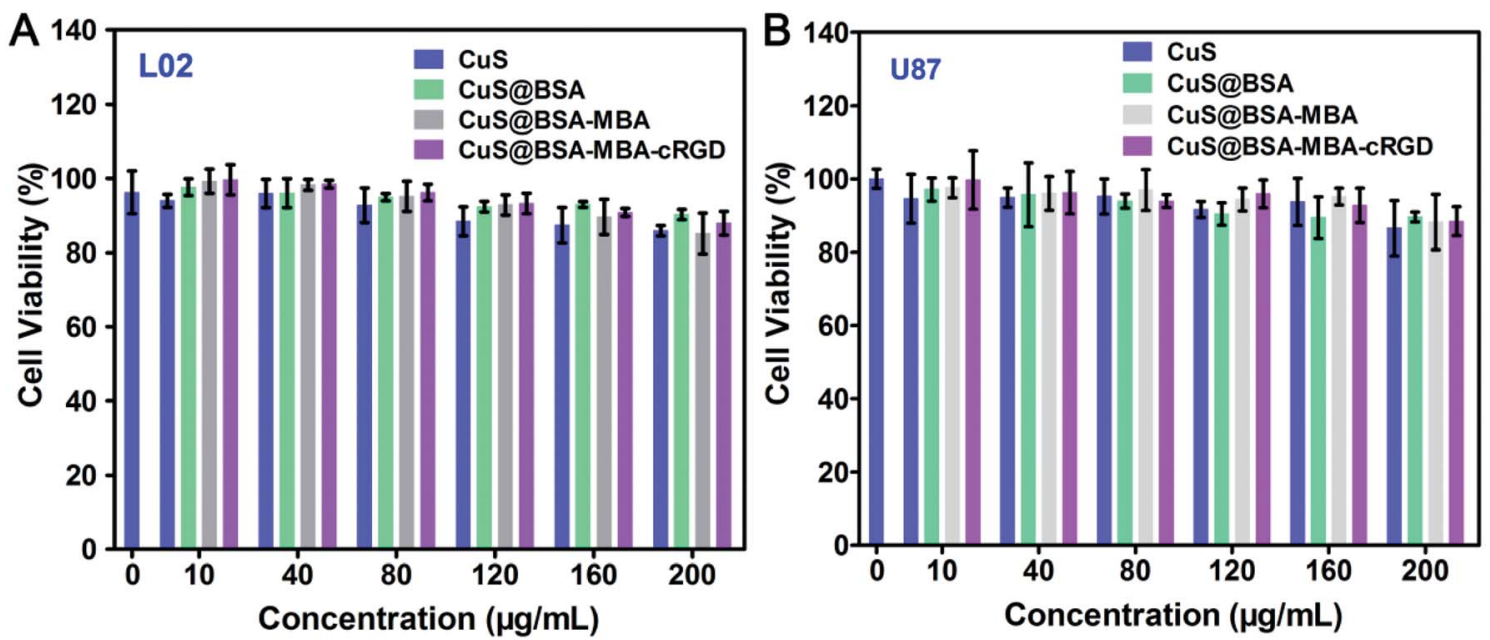

Fig. 7 The cytotoxicity evaluation of CuS NPs, CuS@BSA, CuS@BSA-MBA and CuS@BSA-MBA-cRGD on (A) L02 cell line and (B) U87MG cell line.

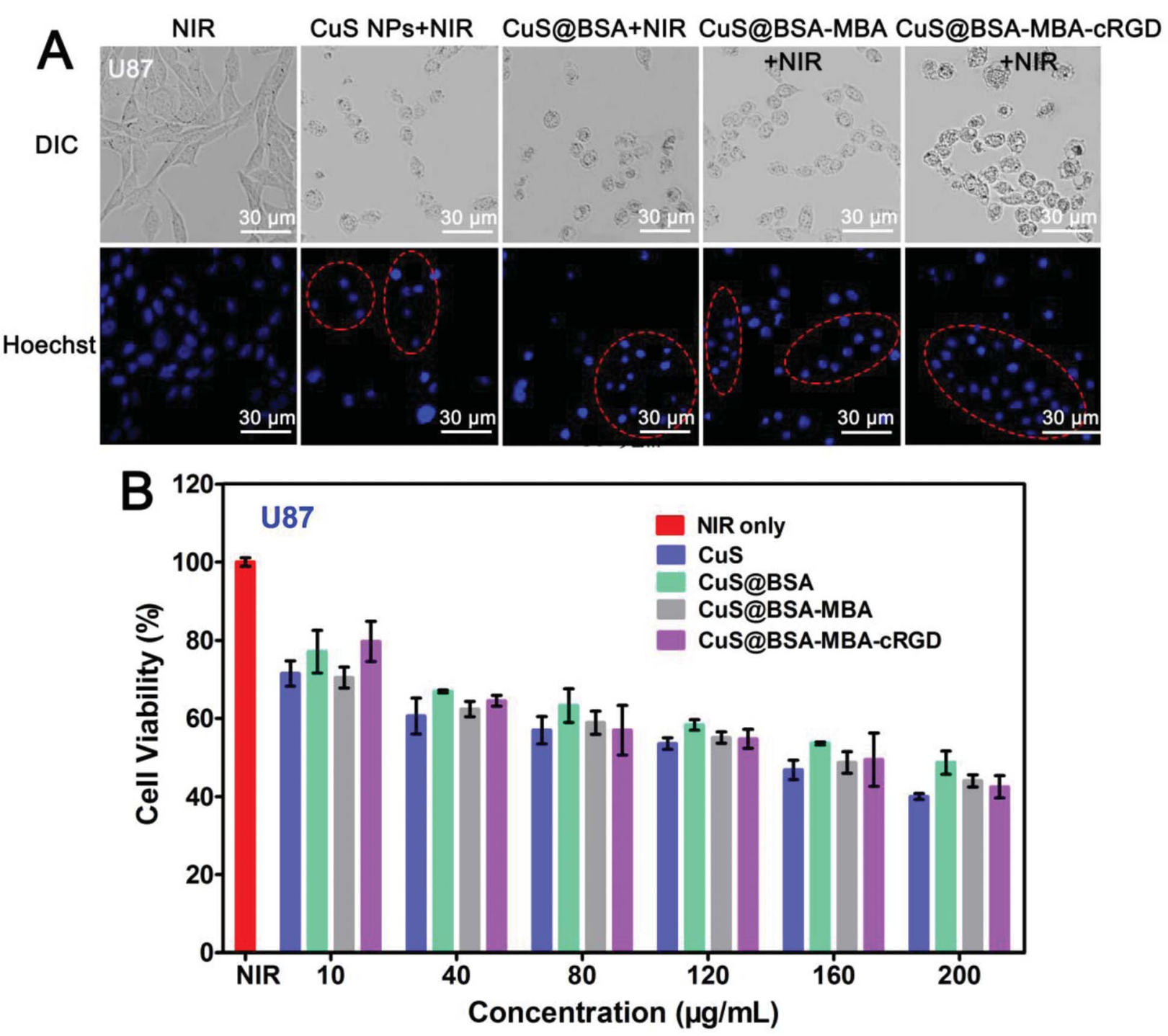

Fig. 8 (A) Morphological images of U87MG cell after incubation with different samples (CuS NPs, CuS@BSA, CuS@BSA-MBA and CuS@BSAMBA-cRGD) and * irradiation by NIR laser $\left(808 \mathrm{~nm}, 1.0 \mathrm{~W} \mathrm{~cm}^{-2}\right.$ ) for $5 \mathrm{~min}$; (B) quantitative analysis of CuS NPs, CuS@BSA, CuS@BSA-MBA and CuS@BSA-MBA-cRGD on the growth inhibition of U87MG cells at different concentrations $\left(10,40,80,120,160,200 \mu \mathrm{g} \mathrm{mL}{ }^{-1}\right)$ after irradiated by NIR laser for $5 \mathrm{~min}$, the NIR group was irradiated by $808 \mathrm{~nm}$ only and served as a control group. 
around $802 \mathrm{~nm}$ and the effective conversion of NIR light to heat. And the fall is due to the photobleaching of the probe after extension of the irradiation time followed by the decreased photothermal conversion efficiency. Fig. 6B indicates that the temperature of CuS NPs increases dramatically as the irradiation time extends. And the temperature curves are concentration dependent. The photothermal variation curves of CuS@BSA (Fig. 6C) show that the temperature increase is not as obvious as CuS NPs at the same experimental condition. The possible mechanism is that BSA coupled to the surface of CuS NPs blocks the irradiation light in some extent, causing CuS@BSA slightly attenuated compared to CuS NPs. CuS@BSA-
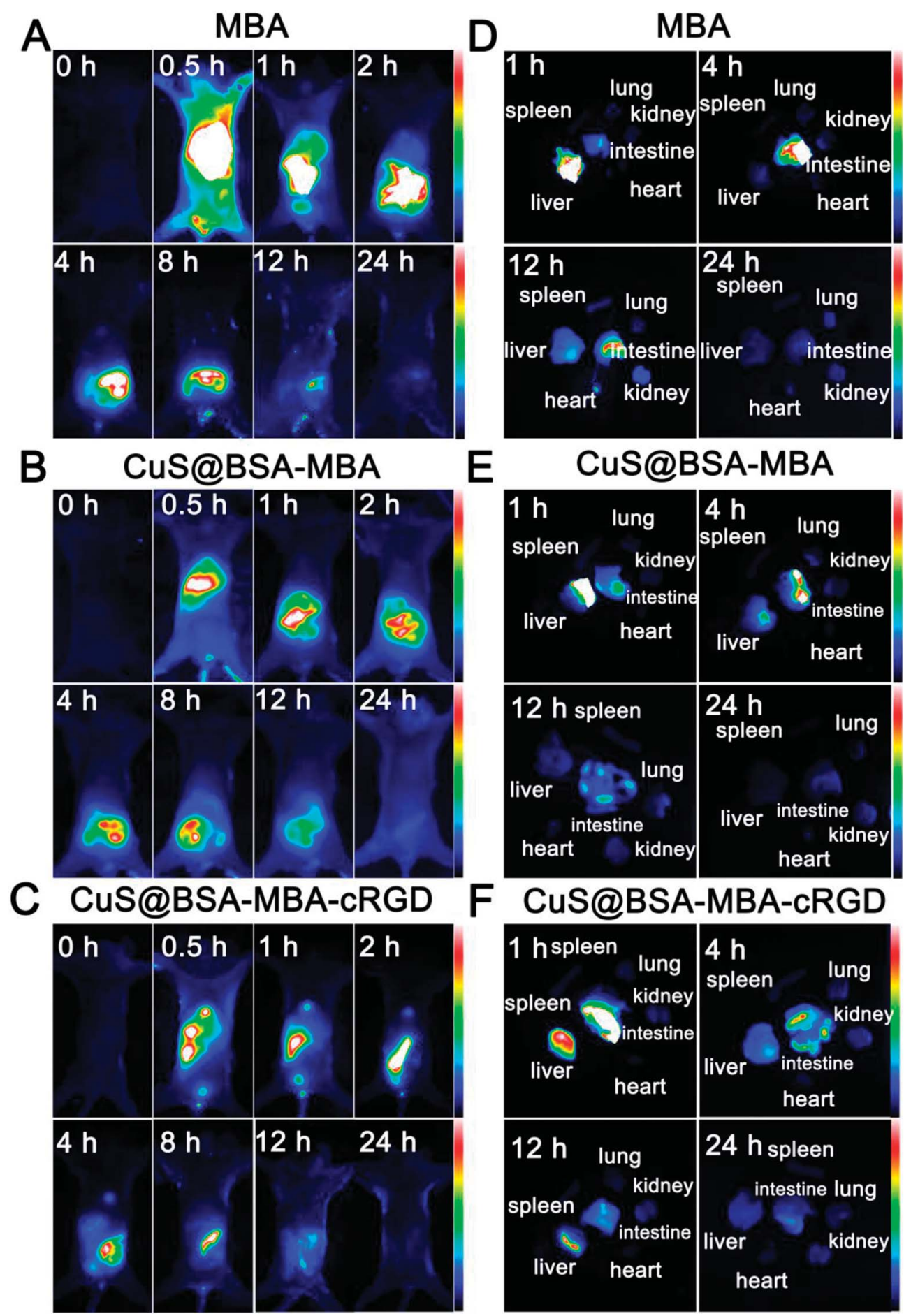

Fig. 9 Dynamic distribution of (A) MBA, (B) CuS@BSA-MBA and (C) CuS@BSA-MBA-cRGD in normal mice monitored by NIR fluorescence imaging system; the ex vivo fluorescence images of isolated organs (heart, liver, spleen, lung, kidney and intestine) from the normal mice at $1 \mathrm{~h}$, $4 \mathrm{~h}, 12 \mathrm{~h}$ and $24 \mathrm{~h}$ post-injection of (D) MBA, (E) CuS@BSA-MBA and (F) CuS@BSA-MBA-cRGD. 
MBA and CuS@BSA-MBA-cRGD have the obvious photothermal effect compared to CuS@BSA at the same mass (Fig. 6D and E), which can be attributed to the compensation of NIR dye MBA. Moreover, CuS@BSA-MBA-cRGD (Fig. 6E) has similar photothermal effect as CuS@BSA-MBA. As shown in Fig. 6E, the temperature rise profile of CuS@BSA-MBA-cRGD is concentration dependent. For the different concentrations of CuS@BSAMBA-cRGD aqueous solutions $\left(0.53,1.05,2.1,4.2 \mathrm{mg} \mathrm{mL}^{-1}\right)$, the temperature increased from 17.9 to $62.5{ }^{\circ} \mathrm{C}$ after 6 minutes of irradiation. At the concentration of $0.5 \mathrm{mg} \mathrm{mL}^{-1}$, a temperature rise of over $36.3{ }^{\circ} \mathrm{C}$ was observed after 2 min of irradiation. At a higher concentration of $4.2 \mathrm{mg} \mathrm{mL}^{-1}$, the temperature increased $49.3^{\circ} \mathrm{C}$. From the semi-quantitative analysis in Fig. $6 \mathrm{~F}$ and $\mathrm{G}$, it is easily to find out that the nanocomposites based on CuS NPs all exhibited efficient photothermal effect at different concentrations $\left(4.2 \mathrm{mg} \mathrm{mL} \mathrm{m}^{-1}\right.$ and $200 \mu \mathrm{g} \mathrm{mL} \mathrm{mL}^{-1}$ ). The above results indicated that the photothermal agent CuS@BSA-MBAcRGD has a favorable photothermal conversion efficiency and it is a promising candidate for PTT.

\subsection{Cytotoxicity of CuS@BSA-MBA-cRGD in vitro}

To evaluate the biocompatibility of CuS@BSA-MBA-cRGD for further biological application, the toxicity of CuS NPs and their nanocomposites were investigated on U87MG tumor cells and L02 normal cells by MTT assessments. As shown in Fig. 7, after $24 \mathrm{~h}$ of incubation, the cell survival rate of CuS NPs, CuS@BSA, CuS@BSA-MBA and CuS@BSA-MBA-cRGD are all above 85\%, which indicates that all these nanocomposites have good biocompatibility on the cellular level and could be used for further in vivo experiments.

Chen et al. developed water-soluble CuS NPs capped with mesoporous silica (CUS@MSN) as promising new theranostic agents. ${ }^{24}$ Although a long-term toxicity study with a large dose of CuS@MSN showed only minor in vivo toxicity, the biodegradation of MSN is still a prominent issue. Inspired by biomineralization, many NPs have been successfully synthesized by biomimetic synthesis methods using different proteins as templates for feasible clinical translation. Herein, BSA as a classic endogenous protein was selected as the templates to prepare CuS@BSA-MBA-cRGD, and the results verified the favorable biocompatibility of this NP for further biological applications.

\subsection{Anticancer effect of CuS@BSA-MBA-cRGD in vitro}

As shown in Fig. 8A, U87MG cells in the NIR group are in full normal shape and no apoptosis was observed. However, the other four groups of the cells, there are obviously characterized with cell deformation and cell shrinkage (as shown in the red circle in Fig. 8A). In addition, the cytotoxicity of CuS@BSA-MBAcRGD was also quantitatively determined by MTT assay (Fig. 8B). The results indicated that these samples (CuS NPs, CuS@BSA, CuS@BSA-MBA and CuS@BSA-MBA-cRGD) showed dose-dependent antitumor activity. At the highest dose level $\left(200 \mu \mathrm{g} \mathrm{mL}^{-1}\right)$, CuS NPs + NIR had the best inhibitory effect (60\%), and CuS@BSA-MBA-cRGD + NIR was slightly lower (58\%). Thus, it is proved that CuS@BSA-MBA-cRGD has an effective inhibition of tumor cell growth.

\subsection{Biodistribution of CuS@BSA-MBA-cRGD in vivo}

Fig. 9A-C displays the dynamic distribution of MBA, CuS@BSAMBA and CuS@BSA-MBA-cRGD in normal mouse models at $0.5 \mathrm{~h}, 1 \mathrm{~h}, 2 \mathrm{~h}, 4 \mathrm{~h}, 8 \mathrm{~h}, 12 \mathrm{~h}$ and $24 \mathrm{~h}$ post-injection. Compared to the fluorescence signal of MBA appeared in the whole body of the normal mice at $0.5 \mathrm{~h}$, the fluorescence only appeared in the

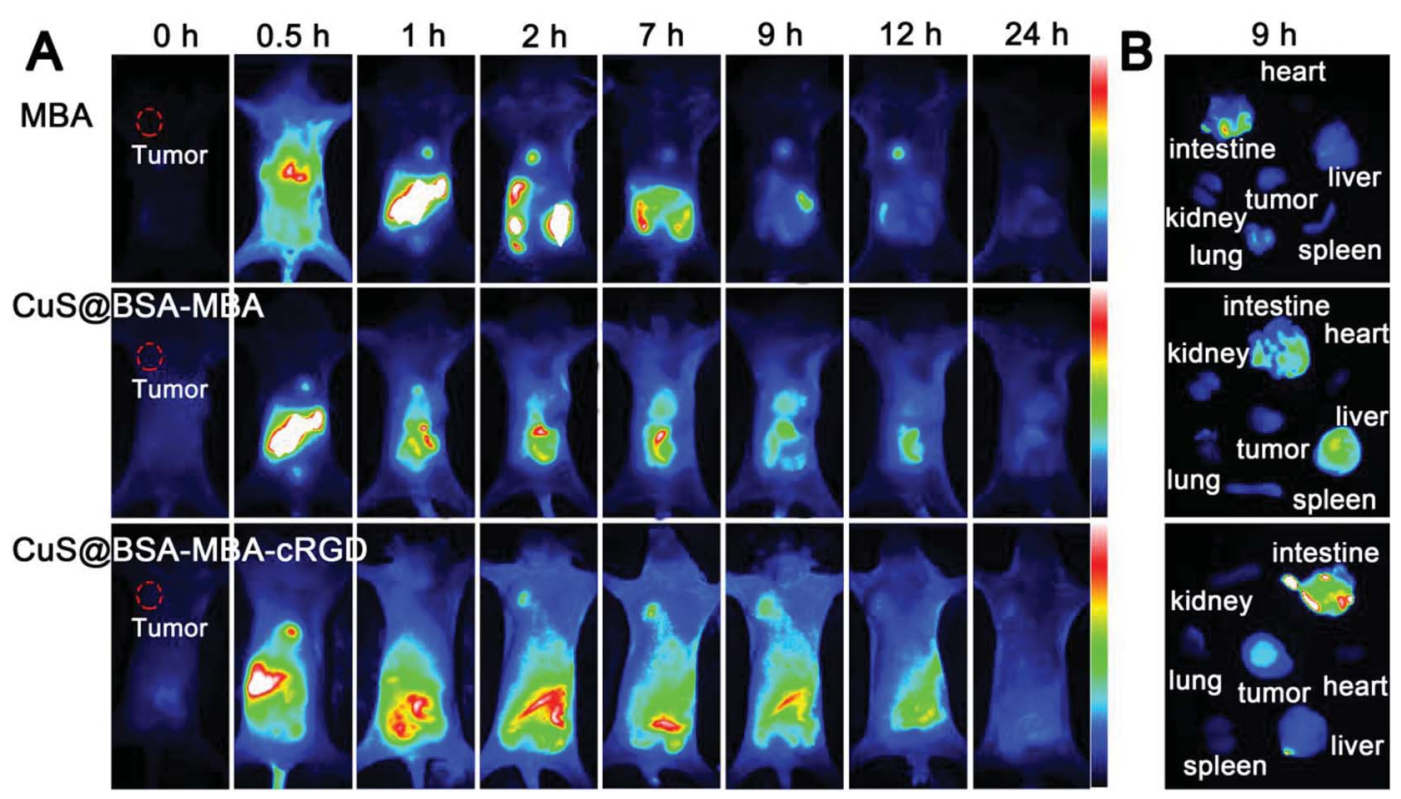

Fig. 10 (A) The in vivo fluorescence images of MBA, CuS@BSA-MBA and CuS@BSA-MBA-cRGD in EAC tumor-bearing mice model within 24 h; (B) the fluorescence images of isolated organs (tumor, heart, liver, spleen, lung, kidney and intestine) from the tumor-bearing mice at $9 \mathrm{~h}$ postinjection of MBA, CuS@BSA-MBA and CuS@BSA-MBA-cRGD. 


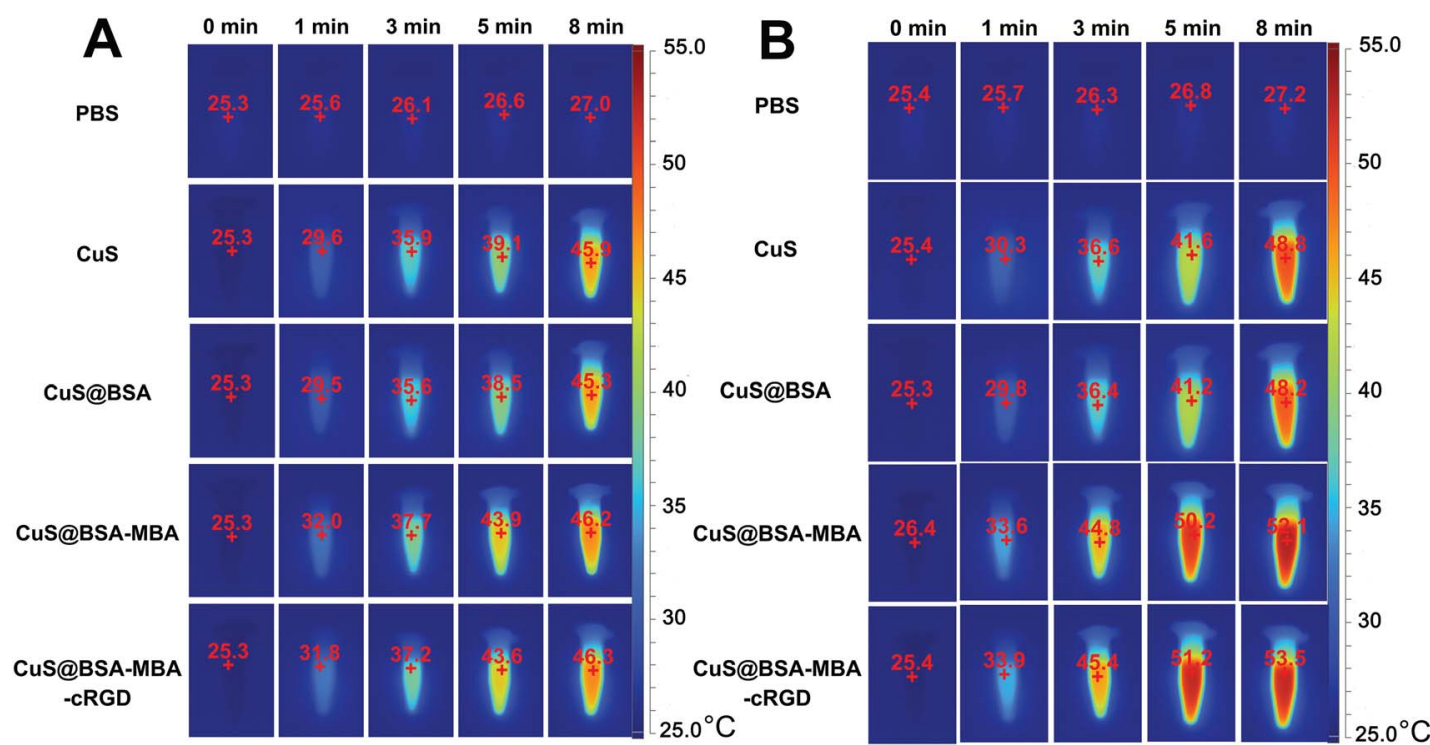

Fig. 11 The infrared thermal images of PBS, CuS NPs, CuS@BSA, CuS@BSA-MBA and CuS@BSA-MBA-cRGD aqueous solution after $808 \mathrm{~nm}$ laser light irradiated for different times $(0,1,3,5$ and $8 \mathrm{~min})$ at different concentrations (A) $200 \mu \mathrm{g} \mathrm{mL} \mathrm{m}^{-1} \mathrm{and}(\mathrm{B}) 750 \mu \mathrm{g} \mathrm{mL}$.

mouse liver at $0.5 \mathrm{~h}$ after tail vein injection of CuS@BSA-MBA and CuS@BSA-MBA-cRGD, which demonstrated that the nanocomposites gradually appeared at the intestinal site of the mice and disappeared after $24 \mathrm{~h}$, which is completely different from the dynamic distribution of MBA. The results also suggested that CuS@BSA-MBA-cRGD excreted from the mice through the liver-intestinal metabolism pathway. ${ }^{25}$

In order to confirm the in vivo result of the nanocomposites, the mice were dissected and the main organs (heart, liver, spleen, lung, kidney and intestine) were collected and imaged at $1 \mathrm{~h}, 4 \mathrm{~h}, 12 \mathrm{~h}$ and $24 \mathrm{~h}$ (Fig. 9D-F). The results are consistent with the vivo imaging. The fluorescence signal of CuS@BSA-
MBA and CuS@BSA-MBA-cRGD first appeared in liver and then transferred to intestine, and finally disappeared after $24 \mathrm{~h}$. It is concluded that the dynamic distribution of CuS@BSA-MBA and CuS@BSA-MBA-cRGD in normal mice is liver-intestinal metabolism pathway.

\subsection{Tumor-targeting ability of CuS@BSA-MBA-cRGD in vivo}

EAC tumor-bearing mice were used to evaluate the tumor specificity of MBA, CuS@BSA-MBA and CuS@BSA-MBA-cRGD. As shown in Fig. 10A, the fluorescence signal of MBA and CuS@BSA-MBA appeared in the whole body of the tumor-

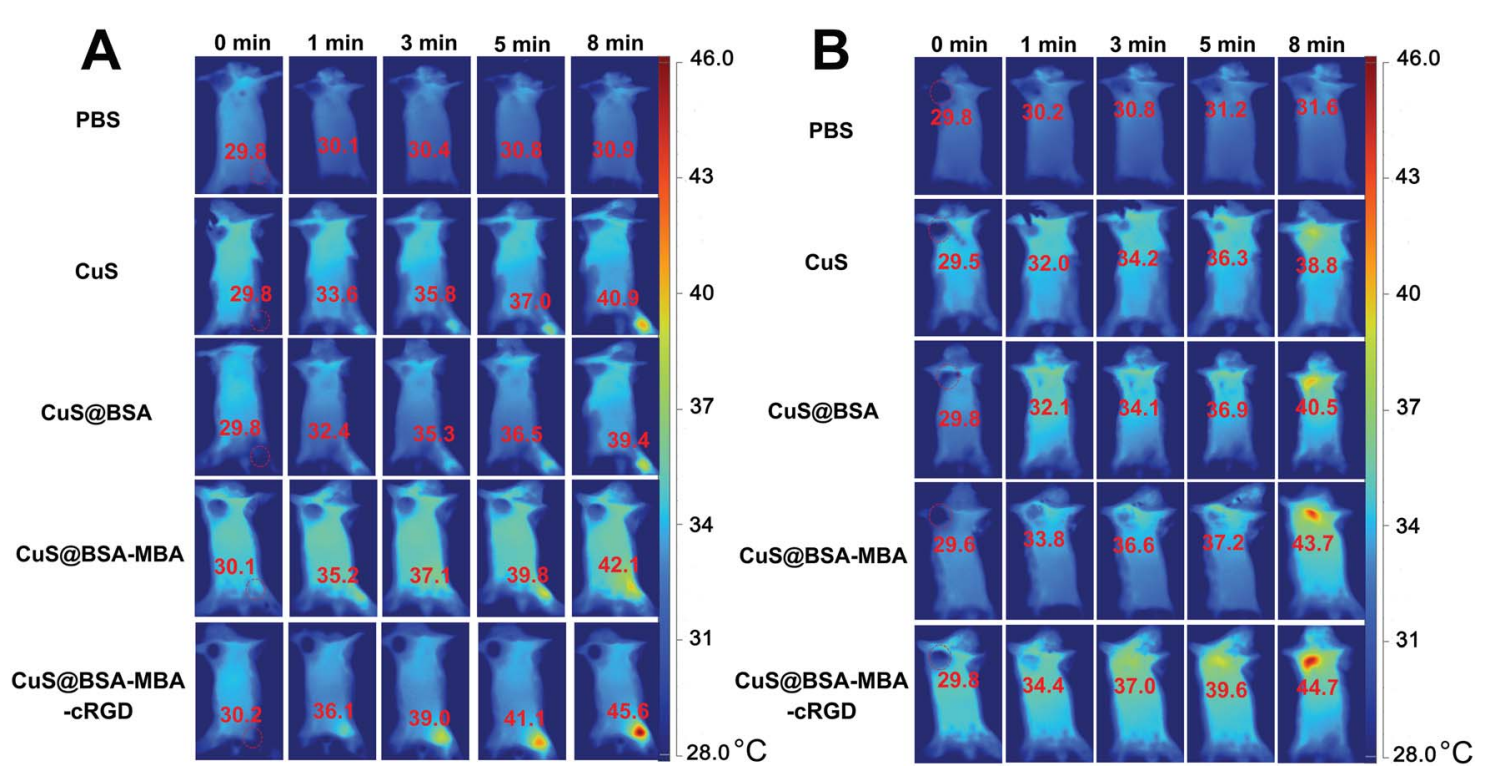

Fig. 12 The infrared thermal images of (A) the normal mice and (B) EAC tumor-bearing mice injected with the PBS, CuS NPs, CuS@BSA, CuS@BSA-MBA and CuS@BSA-MBA-cRGD. 


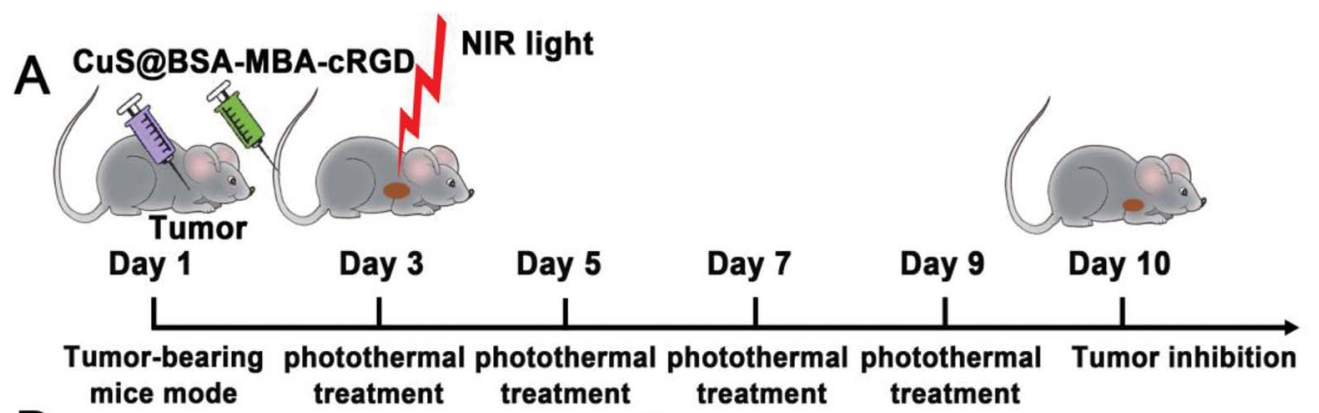

B mice mode treatment treatmen
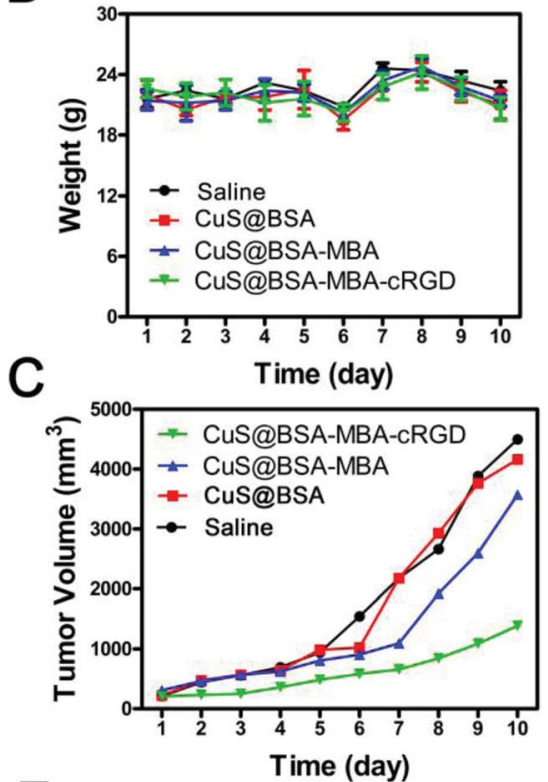

E

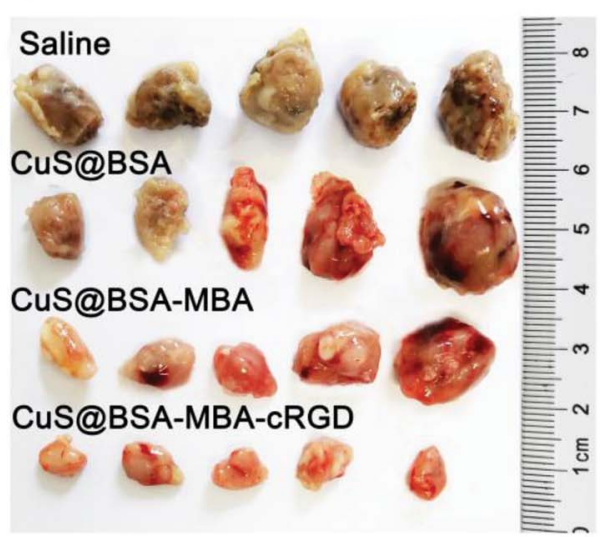

D

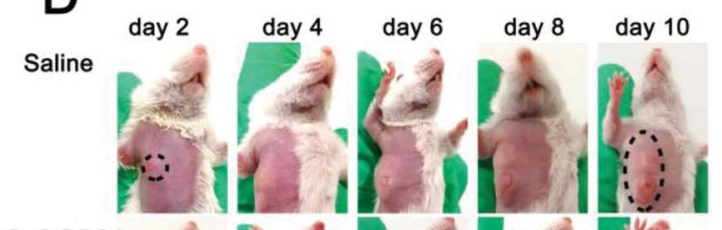

Cus@BsA
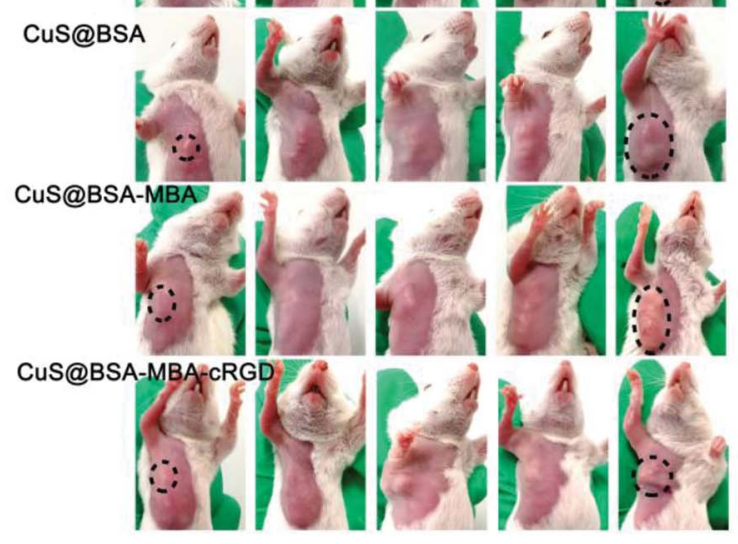

F
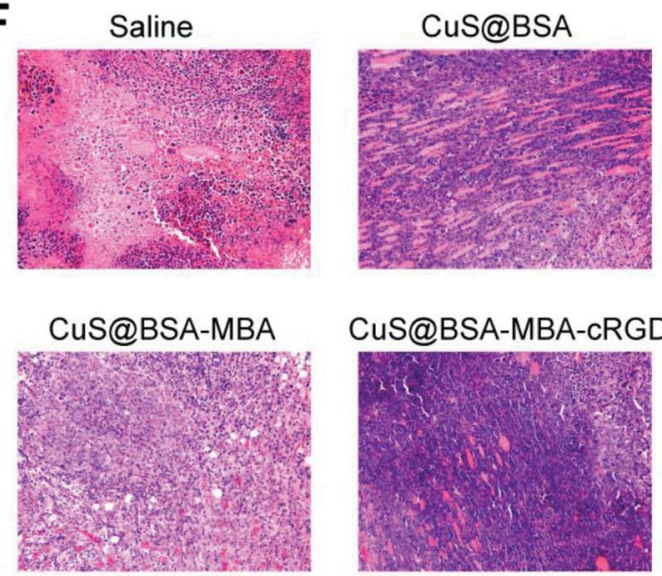

CuS@BSA-MBA-cRGD

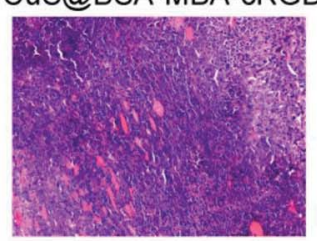

spleen

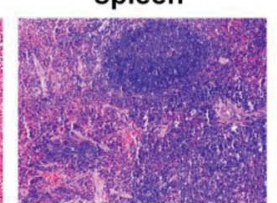

lung

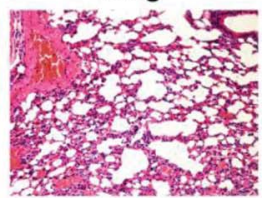

kidney

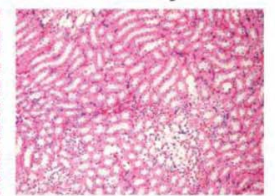

Fig. 13 (A) The experimental scheme to evaluate the anti-tumor effect of CuS@BSA-MBA-cRGD in vivo; (B) body weight and (C) tumor volume of tumor-bearing mice during 10 days of treatment; (D) representative photos of tumor changes and (E) tumor images isolated from tumorbearing mice in different groups after treatment of saline, CuS@BSA + light, CuS@BSA-MBA + light, CuS@BSA-MBA-cRGD + light; the histological images of (F) tumors and (G) the main organs (heart, liver, spleen, lung and kidney) of the tumor-bearing mice after treatment. 
bearing mice after $0.5 \mathrm{~h}$ injection. However, MBA and CuS@BSAMBA did not reach tumor sites efficiently because no visible fluorescence signal was shown in these two groups. For CuS@BSA-MBA-cRGD group, the fluorescence signal initially spread over the liver and intestine of tumor-bearing mice body at $0.5 \mathrm{~h}$ post-injection. At $2 \mathrm{~h}$ post-injection of CuS@BSA-MBAcRGD, it displayed weak fluorescence in tumor, and the fluorescence signal reached peak intensity at $9 \mathrm{~h}$ and totally disappeared at $24 \mathrm{~h}$ post-injection (Fig. 10A). The in vitro fluorescence images of the main organs from MBA, CuS@BSA-MBA and CuS@BSAMBA-cRGD at $8 \mathrm{~h}$ post-injection are shown in Fig. 10B, and the results are consistent with the in vivo imaging. Obviously, this result proved that CuS@BSA-MBA-cRGD has the active tumortargeting capability due to the acceptor-ligand affinity, which is attributed to the potential of cRGD ligands on the NPS to induce integrin-mediated active transport specifically. Overall, it is reasonable to conclude that the cRGD can enter the tumor sites via active transport-mediated pathways.

\subsection{Infrared thermal imaging}

The infrared thermal images of PBS, CuS NPs, CuS@BSA, CuS@BSA-MBA and CuS@BSA-MBA-cRGD aqueous solution after $808 \mathrm{~nm}$ laser light irradiation for different times $(0,1,3,5$ and $8 \mathrm{~min}$ ) or different concentrations (200 and $750 \mu \mathrm{g} \mathrm{mL} \mathrm{m}^{-1}$ ) are shown in Fig. 11. After 1 min of irradiation, the temperatures of all the solutions were still below $40{ }^{\circ} \mathrm{C}$. In comparison, a more significant temperature increase was noticed for the CuS@BSA-MBA and CuS@BSA-MBA-cRGD solutions irradiated for more than $3 \mathrm{~min}$, reaching temperatures of $37-54{ }^{\circ} \mathrm{C}$ throughout the irradiation period. After $5 \mathrm{~min}$ of irradiation, CuS@BSA-MBA (750 $\left.\mu \mathrm{g} \mathrm{mL}^{-1}\right)$ and CuS@BSA-MBA-cRGD (750 $\mu \mathrm{g} \mathrm{mL}{ }^{-1}$ ) solutions reached a temperature higher than $50{ }^{\circ} \mathrm{C}$, and the solutions' temperature was maintained around this temperature even they were irradiated up to $8 \mathrm{~min}$.

As shown in Fig. 12, the region of normal mice injected with the CuS@BSA-MBA and CuS@BSA-MBA-cRGD showed an obvious yellow color, and these regions enlarged with a strong red color $\left(45.6^{\circ} \mathrm{C}\right)$ while the irradiation time was extended from $1 \mathrm{~min}$ to $8 \mathrm{~min}$, while the region injected with PBS still showed blue color after irradiation with the NIR light for $8 \mathrm{~min}$ (Fig. 12A). Fig. 12B showed that the areas of intratumoral injection with CuS@BSA-MBA and CuS@BSA-MBA-cRGD were heated to $44.7^{\circ} \mathrm{C}$ after $8 \mathrm{~min}$ of irradiation but there was no obvious temperature change in the tumor injected with PBS. The results indicated that the as-developed CuS@BSA-MBAcRGD can be utilized as an efficient photothermal agent for tumor ablation as well as a promising infrared thermal imaging contrast agent for infrared thermal imaging.

\subsection{Evaluation of tumor inhibition of CuS@BSA-MBA-cRGD in vivo}

The experimental scheme to evaluate the anti-tumor effect of CuS@BSA-MBA-cRGD + NIR light in EAC tumor-bearing mouse models was shown in Fig. 13A. The weight and tumor volume of tumor-bearing mice were monitored every day, and the changes in body weight of tumor-bearing mice was displayed in Fig. 13B.
No death were observed in the mice during the treatment. In addition, the tumor volume of saline group and CuS@BSA group showed an obvious increase after 6 days of treatment. The tumor growth of CuS@BSA-MBA + NIR light group was not as fast as that for saline group and the CuS@BSA group but significantly faster than CuS@BSA-MBA-cRGD + NIR light group (Fig. 13C). The tumor inhibition rate of CuS@BSA-MBA-cRGD + NIR light was $49.1 \%$. All the results showed that the tumor inhibition effect of CuS@BSA-MBA-cRGD + NIR light was much better than CuS@BSA-MBA + NIR light (Fig. 13D and E). Furthermore, the tumor-inhibition effect and biotoxicity of CuS@BSA-MBA + NIR light and CuS@BSA-MBA-cRGD + NIR light on tumor-bearing mice were also assessed by histological analysis. As shown in Fig. 13F, the tumor cells loosely arranged and appeared vacuoles with nuclear enlargement, nuclear deformity, nuclear and cytoplasmic abnormalities after treatment of CuS@BSA-MBA-cRGD + NIR light. As shown in Fig. 13G, there are no obvious pathological changes for different organs (heart, liver, lung and kidney) except an unobvious damage of spleen for CuS@BSA-MBA-cRGD + NIR light group. It is concluded that CuS@BSA-MBA-cRGD irradiated by NIR light has an effective tumor inhibition and it is a promising candidate for targeting photo-thermal therapy.

It is worth to note that MBA conjugated PTT agents (CuS@BSA-MBA) improve the tumor inhibition efficacy on the basis of increase of light absorbance efficiency, thus enhance the PTT effects. Furthermore, cRGD conjugated CuS@BSA-MBA (CuS@BSA-MBA-cRGD) further improve the tumor therapy efficacy due to the synergistic effects of tumor-targeting delivery and favorable PTT effects.

\section{Conclusion}

In summary, a diagnostic and tumor-targeting nanocomposite (CuS@BSA-MBA-cRGD) with photothermal effect was successfully designed and synthesized. Through the use of MBA and cRGD, we can directly observe the tumor-bearing mouse model of CuS@BSA-MBA-cRGD biodistribution, and locate the tumor site accurately. The tumor cells displayed prominent morphological changes and apoptosis after CuS@BSA-MBA-cRGD treatment. Furthermore, intensify tumor treatment effect of CuS@BSA-MBA-cRGD was also demonstrated in the tumorbearing mouse model. This present study provides a good platform for diagnosis and treatment, and it is expected to prompt further exploration of the active target efficiency to achieve better tumor treatment.

\section{Conflicts of interest}

There are no conflicts to declare.

\section{Acknowledgements}

The authors are grateful to the Natural Science Foundation Committee of China (NSFC 81671803), a project supported by the National Key Research and Development Program (Grant No. 2017YFC0107700), the Outstanding Youth Foundation of 
Jiangsu Province (GX20171114003) and funded by the Priority Academic Program Development of Jiangsu Higher Education Institutions for their financial support.

\section{Notes and references}

1 B. Pang, Y. Zhao, H. Luehmann, et al., (64) Cu-Doped PdCu@Au Tripods: A Multifunctional Nanomaterial for Positron Emission Tomography and Image-Guided Photothermal Cancer Treatment, ACS Nano, 2016, 10(3), 3121-3131.

2 M. R. KAli, I. M. Ibrahim, H. R. Ali, et al., Treatment of natural mammary gland tumors in canines and felines using gold nanorods-assisted plasmonic photothermal therapy to induce tumor apoptosis, Int. J. Nanomed., 2016, 11, 4849-4863.

3 R. Vankayala, C. C. Lin, P. Kalluru, et al., Gold nanoshellsmediated bimodal photodynamic and photothermal cancer treatment using ultra-low doses of near infra-red light, Biomaterials, 2014, 35(21), 5527.

4 C. Yan, Q. Tian and S. Yang, Recent advances in the rational design of copper chalcogenide to enhance the photothermal conversion efficiency for the photothermal ablation of cancer cells, RSC Adv., 2017, 7(60), 37887-37897.

5 Z. Zha, S. Zhang, Z. Deng, et al., Enzyme-responsive copper sulphide nanoparticles for combined photoacoustic imaging, tumor-selective chemotherapy and photothermal therapy, Chem. Commun., 2013, 49(33), 3455-3457.

6 K. Wang, Y. Zhang, J. Wang, et al., Self-assembled IR780loaded transferrin nanoparticles as an imaging, targeting and PDT/PTT agent for cancer therapy, Sci. Rep., 2016, 6, 27421.

7 G. Gollavelli and Y. C. Ling, Magnetic and fluorescent graphene for dual modal imaging and single light induced photothermal and photodynamic therapy of cancer cells, Biomaterials, 2014, 35(15), 4499-4507.

8 Z. Meng, F. Wei, R. Wang, et al., NIR-Laser-Switched in vivo Smart Nanocapsules for Synergic Photothermal and Chemotherapy of Tumors, Adv. Mater., 2016, 28(2), 245.

9 Z. Meng, F. Wei, W. Ma, et al., Design and Synthesis of "Allin-One" Multifunctional $\mathrm{FeS}_{2}$ Nanoparticles for Magnetic Resonance and Near-Infrared Imaging Guided Photothermal Therapy of Tumors, Adv. Funct. Mater., 2016, 26(45), 8231.

$10 \mathrm{~S}$. Goel, F. Chen and W. Cai, Synthesis and biomedical applications of copper sulfide nanoparticles: from sensors to theranostics, Small, 2014, 10(4), 631-645.

11 P. Møller, D. V. Christophersen, N. R. Jacobsen, et al., Atherosclerosis and vasomotor dysfunction in arteries of animals after exposure to combustion-derived particulate matter or nanomaterials, Crit. Rev. Toxicol., 2016, 46(5), 1.
12 H. Maeda, The link between infection and cancer: tumor vasculature, free radicals, and drug delivery to tumors via the EPR effect, Cancer Sci., 2013, 104(7), 779-789.

13 Q. Zhao, X. Yi, M. Li, et al., High near-infrared absorbing $\mathrm{Cu}_{5} \mathrm{FeS}_{4}$ nanoparticles for dual-modal imaging and photothermal therapy, Nanoscale, 2016, 8(27), 13368.

14 N. Yu, Y. Hu, X. Wang, et al., Dynamically tuning nearinfrared-induced photothermal performances of $\mathrm{TiO}_{2}$ nanocrystals by $\mathrm{Nb}$ doping for imaging-guided photothermal therapy of tumors, Nanoscale, 2017, 9(26), 9148.

15 J. Sun, R. Gui, H. Jin, et al., CuS nanocrystal@microgel nanocomposites for light-regulated release of dual-drugs and chemo-photothermal synergistic therapy in vitro, $R S C$ Adv., 2016, 6(11), 8722-8728.

16 X. Wang, Q. Zhang, L. Zou, et al., Facile-synthesized ultrasmall CuS nanocrystals as drug nanocarriers for highly effective chemo-photothermal combination therapy of cancer, RSC Adv., 2016, 6(25), 20949-20960.

17 L. Hou, X. Shan, L. Hao, et al., Copper sulfide nanoparticlebased localized drug delivery system as an effective cancer synergistic treatment and theranostic platform, Acta Biomater., 2017, 54, 307.

18 Z. Wang, P. Huang, O. Jacobson, et al., BiomineralizationInspired Synthesis of Copper Sulfide-Ferritin Nanocages as Cancer Theranostics, ACS Nano, 2016, 10(3), 3453-3460.

19 S. Peng, Y. He, M. Er, et al., Biocompatible CuS-based nanoplatforms for efficient photothermal therapy and chemotherapy in vivo, Biomater. Sci., 2017, 5(3), 475.

20 Y. Miura, T. Takenaka, K. Toh, et al., Cyclic RGD-linked polymeric micelles for targeted delivery of platinum anticancer drugs to glioblastoma through the blood-brain tumor barrier, ACS Nano, 2013, 7(10), 8583.

21 Y. Li, W. Lu, Q. Huang, et al., Copper sulfide nanoparticles for photothermal ablation of tumor cells, Nanomedicine, 2010, 5(8), 1161.

22 L. Wang, ChemInform Abstract: Synthetic Methods of CuS Nanoparticles and Their Applications for Imaging and Cancer Therapy, RSC Adv. , 2016, 6, 82596-82615.

23 C. Dan, B. Li, S. Cai, et al., Dual targeting luminescent gold nanoclusters for tumor imaging and deep tissue therapy, Biomaterials, 2016, 100, 1.

24 F. Chen, H. Hong, S. Goel, et al., In vivo Tumor Vasculature Targeting of CuS@MSN Based Theranostic Nanomedicine, ACS Nano, 2015, 9(4), 3926-3934.

25 O. A. Almazroo, M. K. Miah and R. Venkataramanan, Drug Metabolism in the Liver, Clinics in Liver Disease, 2016, 21(1), 1-20. 